\title{
Liquid flow and control without solid walls
}

Peter Dunne ${ }^{1,2 \dagger}$, Takuji Adachi ${ }^{1,3 \dagger}$, Arvind Arun $\operatorname{Dev}^{2}$, Alessandro Sorrenti ${ }^{1,4}$, Lucas Giacchetti ${ }^{1}$, Anne Bonnin ${ }^{5}$, Catherine Bourdon 6 , Pierre H. Mangin 6 , J.M.D. Coey ${ }^{7}$, Bernard Doudin ${ }^{2}$, Thomas M. Hermans $^{1, *}$

Affiliations:

${ }^{1}$ Université de Strasbourg, CNRS, ISIS, 8 allée Gaspard Monge, 67000 Strasbourg, France

${ }^{2}$ Université de Strasbourg, CNRS, IPCMS UMR 7504, 23 rue du Loess, F-67034 Strasbourg, France

${ }^{3}$ Department of Physical Chemistry, Sciences II, University of Geneva, 30, Quai Ernest Ansermet, 1211 Geneva, Switzerland

${ }^{4}$ Institute for Chemical and Bioengineering, Department of Chemistry and Applied Biosciences, ETH Zürich, Vladimir Prelog Weg 1, 8093 Zürich, Switzerland

${ }^{5}$ Paul Scherrer Institut, CH-5232, Villigen PSI, Switzerland

${ }^{6}$ Université de Strasbourg, INSERM, EFS Grand-Est, BPPS UMR-S1255, FMTS, Strasbourg, F-67000, France

${ }^{7}$ School of Physics and CRANN, Trinity College, Dublin 2, Ireland

*Correspondence to: hermans@unistra.fr

${ }^{\dagger}$ Equal contribution

Solid walls become increasingly important when miniaturizing fluidic circuitry. ${ }^{1}$ They limit flowrates achievable for a given pressure drop, and are plagued by fouling ${ }^{2}$. Approaches to reduce the wall interactions include hydrophobic coatings ${ }^{3}$, liquid-infused porous surfaces ${ }^{4-6}$, nanoparticle surfactant jamming ${ }^{7}$, changing the surface electronic structure ${ }^{8}$, electrowetting ${ }^{9,10}$, surface tension pinning ${ }^{11,12}$, and atomically flat channels ${ }^{13}$. A better solution may be to avoid the solid walls altogether. Droplet microfluidics or sheath flow achieves this, but require continuous flow of both the liquid transported and the outer carrier liquid ${ }^{1,14}$. Here we demonstrate a new approach, where wall-less aqueous liquid channels are surrounded by an immiscible magnetic liquid, both being stabilised by a quadrupolar magnetic field. This creates self-healing, uncloggable, anti-fouling, and near-frictionless liquid-in-liquid fluidic channels with millimetre effective slip lengths. Pumping is achieved by moving permanent magnets that have no physical contact with the liquid channel. We show that this magnetostaltic pumping method can be used to transport whole human blood with very little damage due to shear forces; haemolysis is reduced by an order of magnitude compared to traditional peristaltic pumping. Our liquid-inliquid approach provides new avenues to transport delicate liquids, particularly when scaling 
channels down to the micron scale with no need for high pressures, while retaining basic microfluidic circuitry functionalities.

To avoid contact with the walls of a device, magnetic forces can be used to levitate particles or live cells in a paramagnetic liquid ${ }^{15}$. Furthermore, nearly wall-less microfluidic channels have been demonstrated where continuous 'magnetic antitubes' of water surrounded by an aqueous paramagnetic salt solution are stabilised ${ }^{16}$ using an electromagnet and iron tracks. However, the antitube lifetime was limited by ion interdiffusion between the two liquids, the salts were toxic, and contact with one stationary wall containing the track could not be avoided. Here we overcome all these limitations, creating entirely wall-less microfluidic channels consisting of diamagnetic antitubes completely enclosed by immiscible, and in select cases non-toxic paramagnetic fluids.

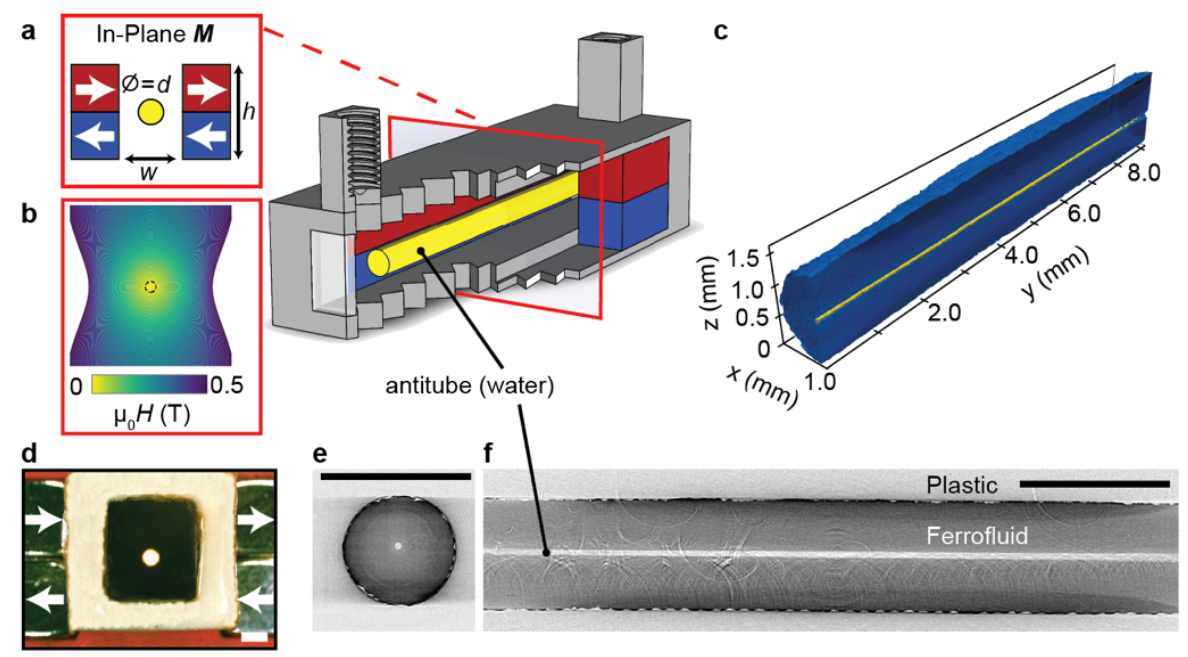

Fig. 1 Wall-less magnetic confinement in a fluidic channel. Centre: exploded view, where permanent magnets (red, blue) in an in-plane quadrupolar configuration create a low-field zone at the centre a, where an antitube of water (yellow) is stabilized inside an immiscible magnetic liquid; $\mathbf{b}$ contour plot of the magnetic field; $\mathbf{c}$ synchrotron X-ray tomographic reconstruction of a water antitube (yellow) with diameter $81 \mu \mathrm{m}$ surrounded by ferrofluid (blue); $\mathbf{d}$ optical end-view of a water antitube in ferrofluid; e X-ray end-view crosssection from tomographic data at $y=4 \mathrm{~mm}$; and $\mathbf{f} X$-ray side-view cross-section from tomographic data at $x=1$ $\mathrm{mm}$. Scale bars (black/white) are $2 \mathrm{~mm}$. 
The key to wall-less magnetic confinement is an extended quadrupolar flux source leading to a null magnetic field along a line at the centre (Fig. 1a,b). Appropriately-magnetised $\mathrm{Nd}_{2} \mathrm{Fe}_{14} \mathrm{~B}$ bars were used to define tubular channels, or else custom-made magnet bilayers were waterjet cut to define more complex fluidic circuitry. The magnetic bars are housed in 3D-printed supports with conventional microfluidic inlet and outlet ports (Fig. 1, and Fig. E1 in Extended Data). The various magnetic fluids used are listed in Table 1; stronger confinement is achieved with ferrofluids, but optical transparency is possible using 'Magoil'. The former are colloidal liquids of magnetite, $\mathrm{Fe}_{3} \mathrm{O}_{4}$, nanoparticles suspended in a carrier fluid, while the latter is a rare-earth based oil, inspired by the diethylenetriaminepentaacetate-based contrast agents used for magnetic resonance imaging ${ }^{17}$ (Fig. E2 and Methods M1, M2). We used commercial ferrofluids from Ferrotec, APG311 and EMG900, 2 vol.\% and 13 vol.\% magnetite in hydrocarbon oils respectively, and from Qfluidics, MKC and MD4 which are 29 vol.\% magnetite in hydrocarbons, and 5 vol.\% magnetite in perfluorodecalin, respectively.

Table 1. Characteristics of the magnetic fluids.

\begin{tabular}{cccc}
\hline Magnetic liquid & Susceptibility $\chi$ & Viscosity $\boldsymbol{\eta}(\mathbf{m P a}$ s)* & Appearance \\
\hline Magoil & $4.7 \times 10^{-4}$ & 600 & Transparent \\
\hline APG311 ferrofluid & 0.151 & 70 & Opaque / black \\
\hline EMG900 ferrofluid & 1.67 & 60 & Opaque / black \\
\hline MKC ferrofluid & 1.88 & 380 & Opaque / black \\
\hline MD4 ferrofluid & 0.315 & 180 & Opaque / black \\
\hline
\end{tabular}

*at a shear rate of $100 \mathrm{~s}^{-1}$

Aqueous antitubes were typically formed by injecting ferrofluid into water-filled quadrupole channels and visualised by X-ray imaging \& tomography (Fig. 1c-e). Tomography images unambiguously reveal the wall-less features, confirmed by visual inspection of transparency of a tubular shape on antitubes up to $1 \mathrm{~m}$ long (Fig. E1g). Optical imaging was found possible through no more than $200 \mu \mathrm{m}$ thick ferrofluid and a high contrast camera (Fig. E3c-g), where we can take advantage of the optical resolution to image the smallest antitube features. Antitubes in transparent Magoil could be 
imaged using standard optical or fluorescent microscopy by adding contrast ink or fluorescent dye to the water antitube, allowing real-time visualisation of antitube extrusion and retraction (video 1). Furthermore, trapped gas bubbles that are often problematic in conventional devices can easily be removed, since their buoyancy in Magoil overcomes the magnetic confinement. For most practical applications, however, ferrofluids are preferred because of their much larger magnetic susceptibility $\chi$ (Table 1); they can withstand significant flow rates while remaining confined in the quadrupole (Fig. $\mathrm{E} 4 \mathrm{a}, \mathrm{b})-\mathrm{a} 1 \mathrm{~mm}$ diameter liquid tube can deliver $\sim 40 \mathrm{~mL} / \mathrm{min}$ flow. In addition, the phase transfer of magnetite nanoparticles into water is very low, with measured iron concentrations mostly below a part per million (Methods M15).
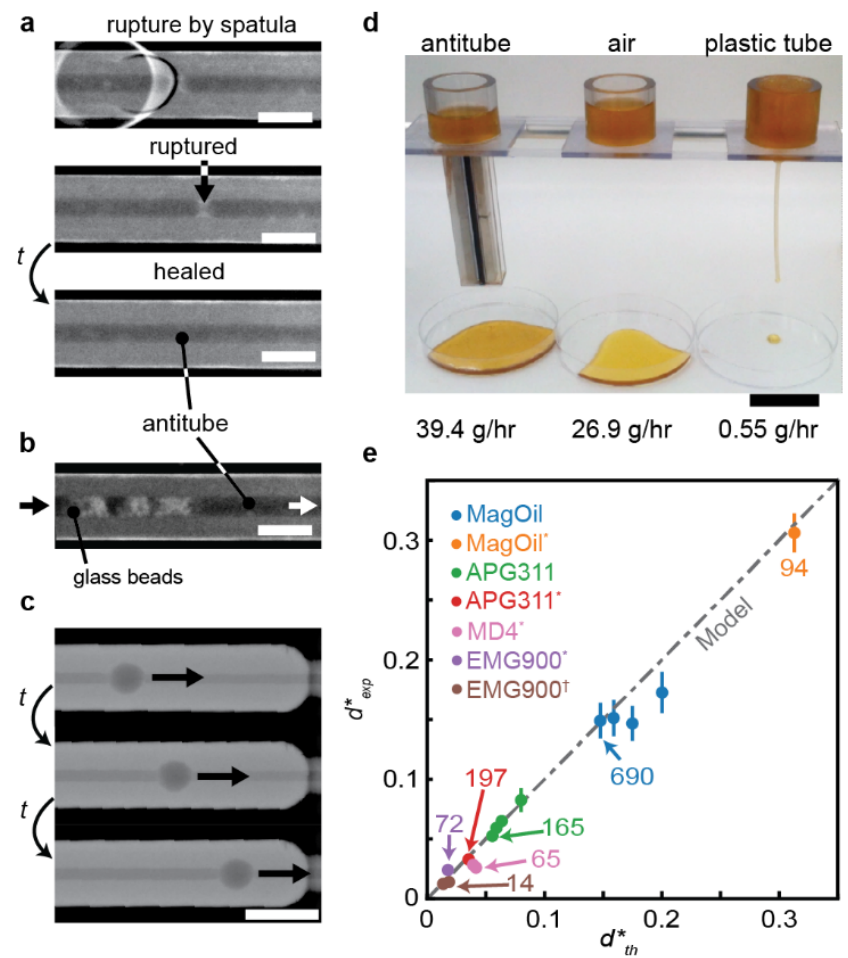

Fig. 2 Unique properties and scaling of water antitubes. a Top-view X-ray images illustrating the mechanical rupture of a static tube using a spatula, followed by self-healing, returning to equilibrium, without being under flow, within minutes; b $0.6 \mathrm{~mm}$ glass beads inserted into a $1.5 \mathrm{~mm}$ antitube that can be expelled with a slight increase in applied pressure of 20 mbar; $\mathbf{c}$ a $2 \mathrm{~mm}$ diameter bead larger than the antitube diameter $(d=0.5 \mathrm{~mm})$ does not cause clogging; there is only a small decrease in flow rate from 30 to $23 \mu 1 \mathrm{~min}^{-1}$ when the bead is present; d comparative flow of honey under gravity (see video 4) through an antitube (left), a normal tube of the same diameter (right) and free fall (centre). Scale bar is $4 \mathrm{~cm}$. e plot of experimental dimensionless antitube diameters 
$d^{*}=d / w$ ( $w$ the spacing between the magnets) versus theoretical values calculated from Eqn. 4 , for a series of magnetic fluids. Numbers indicate the smallest tube diameters in $\mu \mathrm{m}$, and $*$ denotes water containing 1 vol.\% Tween-20, while $\dagger$ denotes 1 vol.\% Span-80 in the ferrofluid in addition to 1 vol.\% Tween-20 in the water. For ferrofluid data each data point represents the average of at least 2 experiments, where at least 5 images were recorded each. For Magoil* at least 10 snapshots were taken from an experimental video. Error bars are standard deviations.

The liquid-in-liquid design offers advantages of stability and robustness for fluid transport. Fig. 2a illustrates self-healing after an antitube in ferrofluid was severed with a spatula. Recovery without applied external pressure is rapid (self-healing in Magoil is illustrated in video 2). The antitubes cannot clog: when glass beads are introduced (Fig. 2b), they are easily flushed out. Even a bead much larger than the antitube diameter can be pushed through using minimal pressure (20 mbar, Fig. 2c). The liquid walls of the antitube stretch to avoid clogging and return to their original size when the obstruction is expelled. A change of external pressure alters the antitube size. In Fig. E4 c,d it can be seen that antitubes remain unchanged with externally applied pressure for an open outlet at atmospheric pressure, but dilate when the same pressure is applied with the outlet closed off. This can also be seen in Fig. 2c where the tube dilates behind the bead to accommodate the increased local pressure. As a demonstration of anti-fouling behaviour, we covalently crosslinked a photoresist inside an antitube, and were able to remove the solid polymer rod from the outlet of the device (Fig. E6 a-c, and video 3).

A further advantage of liquid-in-liquid flow is near-frictionless transport with negligible pressure drop. A dramatic illustration is shown in Fig. 2d and video 4, where flow of a magnetically confined antitube made of honey (dynamic viscosity $\eta_{h}=10$ Pa.s) is compared to honey flow in a standard tube. Here, we observed an antitube flow of $39.4 \pm 0.7 \mathrm{~g} /$ hour, $\sim 70$ times faster than the flow through a conventional plastic tube of the same diameter $d=1.1 \mathrm{~mm},(0.55 \pm 0.10 \mathrm{~g} / \mathrm{hour})$. The ferrofluid acts as a lubricating layer, with an effective slip length $b$ at the honey/ferrofluid boundary that can be approximated by

$$
b=\frac{1}{2}\left[\sqrt{\frac{d^{2}}{2}\left(Q_{N D}+1\right)}-d\right]
$$


where $d$ is the antitube diameter and $Q_{N D}$ is the non-dimensional flow rate given by

$$
Q_{N D}=\frac{128 Q \eta_{h}}{\pi d^{4} \partial P / \partial z}
$$

where $Q$ is the flow rate, and $\partial P / \partial z$ is the pressure gradient in the flow direction (for derivation see methods M18, M19). Experimentally, we obtain an effective slip length of $4.3 \mathrm{~mm}$, meaning that under these conditions the flow is essentially plug-like. Under the assumption of an infinite channel, the pluglike velocity profile can be calculated, as shown analytically in Fig. E4e, f and using numerical methods in Fig. E5a-d, leading to a theoretical effective slip length of $8.1 \mathrm{~mm}$. Remarkably, the flow rate of honey through the antitube was 1.5 times faster than when it fell freely and unconfined, likely due to competition between orifice wetting ${ }^{18}$ and the higher hydrostatic pressure due to the greater height of the honey column in the antitube design.

The stable confinement of an antitube, at equilibrium, results from the competing magnetic energy of the confining fluid and the surface energy $\sigma$ of the magnetic/non-magnetic interface. Inserting these energy densities into the magnetically augmented version of Bernoulli's equation ${ }^{19}$, gives the equilibrium diameter of the antitube, derived in M19:

$$
d=\frac{4 \sigma}{2 \mu_{0} \bar{M} H_{I}+\mu_{0} M_{I}^{2}}
$$

where $H_{I}, M_{I}$ are the magnetic field and magnetization values at the interface, $\mu_{0}$ is the permeability of free space, and $\bar{M}$ is the field-averaged magnetization of the confining fluid. This equation is valid when the magnetic pressure, $1 / 2 \mu_{0} H^{2}$ is significantly larger than any difference in hydrostatic pressure. It can then be linearized when $M=\chi H$, under the geometrical conditions $w \leq 1 / 2 h$, and $d \leq 1 / 2 w$, typical of our devices, where $w$ is the spacing between the magnets. The linear model (LM) gives the minimum equilibrium dimensionless diameter $d^{*}=d / w$ as:

$$
d^{*}=\sqrt[3]{\frac{\pi^{2}}{N_{D} \chi(\chi+1)}}
$$


where $N_{D}=\frac{\mu_{0} M_{r}^{2} w}{\sigma}$ is the magnetic confinement number expressing the ratio of magnetic to surface energies (see M20), and $M_{r}$ is the remnant magnetisation of the permanent magnets. Note that a 1000fold increase in $\chi$ reduces $d^{*}$ by a factor 100 , revealing the importance of the magnetic susceptibility of the confining fluid.

We have studied how the attainable antitube diameters vary with $w$ for the paramagnetic confinement liquids of Table 1. There is good agreement between the experimental points for water antitubes and the predictions of Eqns. (3) and (4), using measured susceptibility $\chi$ and interface energy $\sigma$ (Table E1). The addition of a surfactant to the water phase, the magnetic fluid phase or to both ${ }^{20}$, leads to smaller antitubes since $\sigma$ is lowered; 1 vol. $\%$ or $23 \mathrm{mM} \mathrm{Span}-80$, in the ferrofluid, and 1 vol. $\%$ or $0.58 \mathrm{mM}$ Tween-20 in the aqueous antitube. Although the critical micelle concentrations of Span-80 and Tween20 in water-hydrocarbon interfaces are $1.2 \mathrm{mM}$ and $0.58 \mathrm{mM}$, respectively ${ }^{21}$, we did not observe surfactant-induced structure formation. The close agreement is shown in the plot of dimensionless diameters $d^{*}$ in Fig. 2e where a plot of the experimental values versus predicted diameters collapse onto one curve for all ferrofluids, surface tensions, and magnet gaps used. The smallest antitube that could be detected so far has $d=14 \pm 2 \mu \mathrm{m}$, using a strong ferrofluid, EMG900 (Fig. E3g). Our model predicts that antitubes below $1 \mu \mathrm{m}$ can be stabilised, even with a $100 \mu \mathrm{m}$ sized magnet spacing using a strong ferrofluid (MKC), but they are currently below the detection limit of our imaging methods.

Magnetic confinement can be used to implement basic microfluidic operations. In order to make branched antitube devices we resort to out-of-plane quadrupolar fields, made by waterjet cutting two stacked $\mathrm{Nd}_{2} \mathrm{Fe}_{14} \mathrm{~B}$ plates (Fig. 3a). The null-field line follows the channel centre independently of the channel angle with respect to the magnets, which is not the case for in-plane quadrupoles (not shown). Symmetric splitting of the flow was demonstrated in a ferrofluid antitube Y-junction (video 7). Merging of the flow at a Y-junction was visualized using antitubes stabilized by Magoil. Remarkably, merging and rapid mixing occurs immediately after a Y-junction (Fig. 3b), similar to mixing in magneticallystabilised aqueous paramagnetic tubes surrounded by water ${ }^{16}$. This behaviour is in contrast to the 
laminar flow observed in a 3D printed microfluidic chip with the same channel size and geometry as the antitube (Fig. 3c).

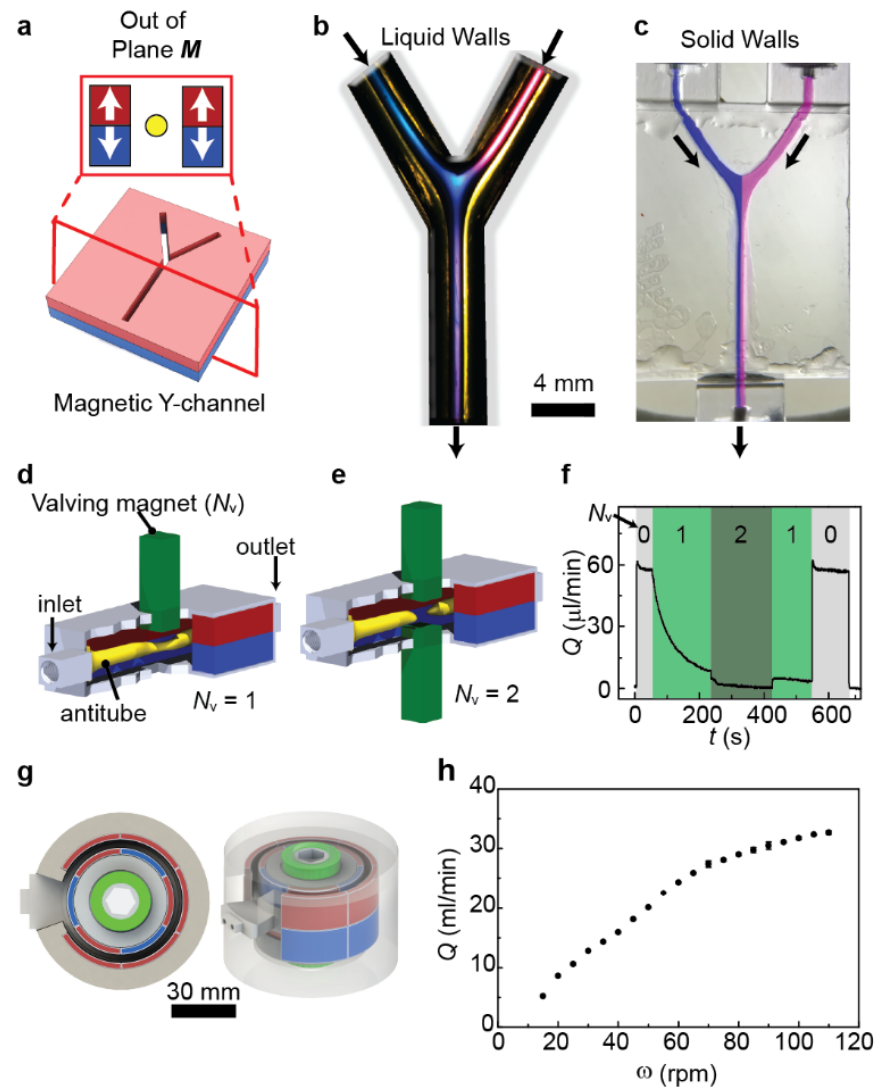

Fig. 3 | Magnetically implemented mixing, valving and pumping fluidic functionalities. a Out of plane magnetization configuration for a Y-junction cut in a double magnet sheet; $\mathbf{b}$ optical image of a $\mathrm{Tb}^{3+}-$ Magoil stabilised aqueous antitube, where blue and pink dye are introduced at the inlets $\left(300 \mu \mathrm{L} \mathrm{min}{ }^{-1}\right)$, and mix immediately upon contact before flowing to the outlet; $\mathbf{c}$ comparison with normal microfluidic channels, where no mixing is observed: $\mathbf{d}$ valving with one magnet $\left(N_{\mathrm{v}}=1\right.$, video 9$)$ or $\mathbf{e}$ two magnets $\left(N_{\mathrm{v}}=2\right.$, video 10$)$; $\mathbf{f}$ measured flow rate under an applied pressure of 100 mbar at the exit port controlled by 1 or 2 valving magnets; $\mathbf{g}$ top-view and isometric-view of a magnetostaltic Qpump with rotating magnetic segments. The orientation of magnetization for the arc segments is radially outward (red) or inward (blue); $\mathbf{h}$ Flow rate of water, $Q$ vs rotation rate $\omega$ of the inner rotor. Error bars represent the standard deviations over 3 samples.

As our microfluidic circuits are magnetically defined, we can exploit the fact that structured magnetic fields can be modulated by mechanical or electrical means to impose unique and versatile control of fluidic devices. Valves can be constructed by moving one or two longitudinally-magnetised bars 
towards the quadrupole axis. The valving magnets simply pinch off the antitube by removing the null field at the centre (Fig. 3d, 3e), thereby imposing a local magnetic pressure, $p=\mu_{0} M H$, and interrupting the liquid flow (see videos 8-10 which plot the calculated $150 \mathrm{mT}$ isovolume surfaces). The burst pressure of these valves was tested with an Elvesys OB1 pressure-driven pump. A single transverse valving magnet could sustain a pressure of 125 mbar, whereas a dual valve (Fig. 3f) withstood 300 mbar. Pumping is an extension of the valving principle; pinch points of magnetic pressure seal off pockets of pumped liquid (e.g. water) which are then displaced by the mechanical actuation of the valving magnets. This can be done by sequential excitation of electro- or electro-permanent magnets, or a rotating array of magnetic spokes (see Fig. E6d-f). A more sophisticated pump based on radially magnetized arc-segments fixed onto a rotor and stator, and a 3D printed fluidic support is illustrated in Fig. 3g, with magnetic field contours shown in Fig. E6j. This 'Qpump', using MKC ferrofluid, produces pressures of up to $900 \mathrm{mbar}$ and flow rates of $32.7 \pm 0.3 \mathrm{~mL} / \mathrm{min}$ (video 12, Fig $3 \mathrm{~h}$ ).

Since the antitube inside the Qpump has no solid walls, we expected that magnetostaltic pumping would be gentler than peristaltic pumping, where a plastic tube is mechanically squeezed by a roller. It is known that blood pumping results in haemolysis, shear-induced rupture of red blood cells that releases haemoglobin. ${ }^{22-26}$ High concentrations of free haemoglobin are cytotoxic, and have been associated with clinical complications including an increased incidence of thrombosis, morbidity, and mortality. ${ }^{22-}$ ${ }^{26} \mathrm{We}$ therefore compared the effects of pumping $6 \mathrm{~mL}$ samples of human donor blood collected on hirudin anticoagulant at $1.5 \mathrm{~mL} / \mathrm{min}$ for $1 \mathrm{~h}$ in a closed loop using either the Qpump or a peristaltic pump (Fig. 4a). Whereas for water sub-ppm transfer of magnetite nanoparticles was observed (cf. M15), for blood $285 \pm 161 \mathrm{ppm}\left(\mu \mathrm{g} \mathrm{Fe} \mathrm{O}_{4} / \mathrm{g}\right.$ blood) was detected (see M17). However, this did not affect haematological parameters such as haematocrit and cell counts of the pumped blood, which were unchanged versus the peristaltic pump (Fig. $4 \mathrm{~b}$ and Table E3). Moreover, functions of platelets which circulated through the Qpump were unchanged as they responded normally to agonists such as thrombin receptor-activating peptides (TRAP) in the widely used light transmission aggregation test (Fig 4b). Further inspection of platelet morphology by scanning electron microscopy (SEM) confirmed that 
platelets were intact and normal after pumping (Fig. E7 a-c). In short, these experiments have revealed no adverse effects of whole blood pumped using the Qpump, as compared to a peristaltic pump (Fig E7d).

In contrast, a large difference was found in the degree of haemolysis (Fig. 4d,e). Platelet rich plasma obtained by centrifugation of whole blood is transparent after pumping using the Qpump, but it is bright red after peristaltic pumping (insets Fig. 4d). Based on the UV-Vis absorbance of haemoglobin ${ }^{27}$ (Fig. 4d), the concentration of plasma-free haemoglobin (PFH) was determined to be 130 $\pm 40 \mathrm{mg} / \mathrm{dL}$ and $12 \pm 5 \mathrm{mg} / \mathrm{dL}$ for the peristaltic pump and Qpump respectively (Fig. 4e). The degree of haemolysis is $11 \pm 6$ times lower for blood pumped by the Qpump versus the traditional peristaltic pump. $\mathrm{PFH}$ values greater than $20 \mathrm{mg} / \mathrm{dL}$ are indicative of significant haemolysis ${ }^{28}$, and previous reports on packed red blood cells transfusion using infusion pumps showed 2-3 orders of magnitude higher PFH values ${ }^{26}$, which indicates that magnetic pumping is a promising way to achieve gentle transportation of blood, or possibly other fragile entities such as antibodies or stem cells. Further studies are planned using a mouse model of extracorporeal membrane oxygenation (ECMO), using a Qpump instead of a peristaltic pump, to investigate survival rates and possible systemic responses upon ferrofluid contact.
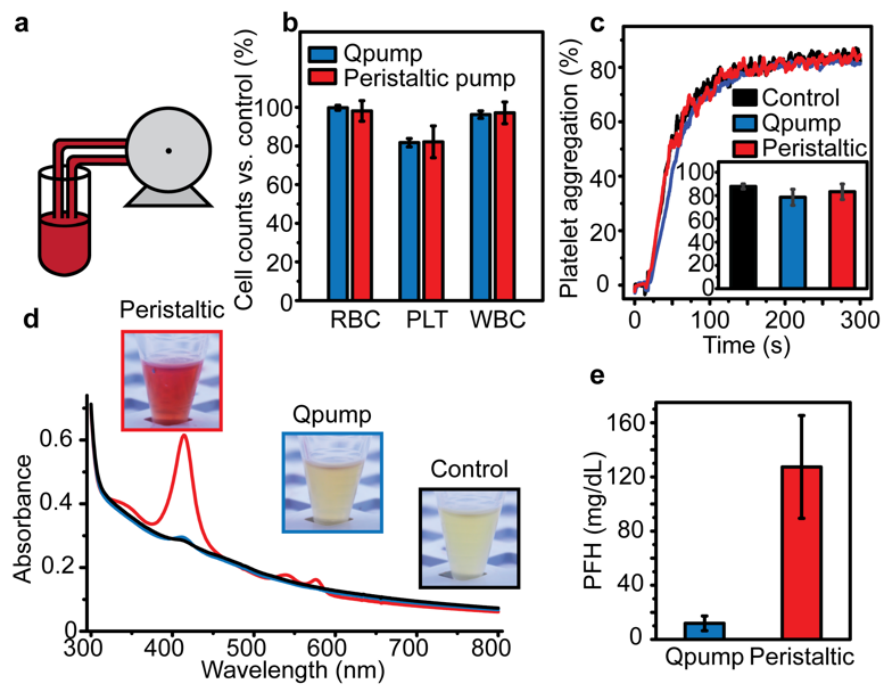
Fig. 4 | Blood pumping by a magnetostaltic pump. Blood was circulated in a closed-loop circuit either by the Qpump (filled with 7x diluted MKC ferrofluid) or a normal peristaltic pump. a Closed-cycle pumping scheme used; b blood cell counts (RBC: red blood cells, PLT: platelets, WBC: white blood cells) after pumping normalized versus control blood; c The degree of platelet aggregation activated by thrombin receptor-activating peptides (TRAP) tested for PRP from blood after peristaltic pumping and Qpumping compared with control blood. Light transmission is minimal before aggregation as platelets scatter light, but upon addition of TRAP the platelets aggregate and transmission increases; d Absorption spectrum of platelet rich plasma (PRP) from blood after peristaltic pumping and Qpumping compared with control blood 25 times diluted with phosphate buffer; e Plasmafree haemoglobin $(\mathrm{PFH})$ concentration estimated from the absorbance of PRP for Qpump and peristaltic pump. Blood was used from three different anonymous donors (cf. Methods section M17), and bars show data from at least 6 measurements. Error bars show standard deviations.

Our extensive investigations have established that magnetic control of liquid-in-liquid flow opens new possibilities for microfluidics, allowing novel channel shapes and low-pressure cargo transport surpassing the current capabilities of standard methods. We have identified the basic physical quantities controlling the size of confined diamagnetic fluid circuitry, and have given examples of key microfluidic device elements, demonstrating the possibilities of this liquid-in-liquid flow technology. The new method promises low-shear flow and pumping, which is of growing importance in biotechnology where delicate cells, proteins, and antibodies are commonly damaged by traditional pumps. ${ }^{29-33}$ If scaled up to 5-7 L/min and combined with a gradient-field separator ${ }^{34}$ to remove traces of transferred magnetite nanoparticles, magnetic blood pumping might be implemented in heart-lung machines during cardiopulmonary bypass surgery, or in devices for extra-corporeal membrane oxygenation. ${ }^{22-25}$ At a more fundamental level, our findings lead us to envisage miniaturized fluidic circuits without solid walls that will be scalable down to the submicron level. We can then take full advantage of the versatility of magnetic control at the nanoscale to open the door to practical low pressure nanofluidics ${ }^{35-37}$.

\section{References:}


1. Tabeling, P. Introduction to Microfluidics. (OUP Oxford, 2005).

2. Mukhopadhyay, R. When Microfluidic Devices Go Bad. Anal. Chem. 77, 429 A-432 A (2005).

3. Zhao, B., Moore, J. S. \& Beebe, D. J. Surface-Directed Liquid Flow Inside Microchannels. Science 291, 1023-1026 (2001).

4. Wong, T.-S. et al. Bioinspired self-repairing slippery surfaces with pressure-stable omniphobicity. Nature 477, 443-447 (2011).

5. Wang, W. et al. Multifunctional ferrofluid-infused surfaces with reconfigurable multiscale topography. Nature 559, 77 (2018).

6. Leslie, D. C. et al. A bioinspired omniphobic surface coating on medical devices prevents thrombosis and biofouling. Nat. Biotechnol. 32, 1134-1140 (2014).

7. Forth, J. et al. Reconfigurable Printed Liquids. Adv. Mater. 1707603 (2018).

8. Secchi, E. et al. Massive radius-dependent flow slippage in carbon nanotubes. Nature 537, 210213 (2016).

9. Banerjee, A., Kreit, E., Liu, Y., Heikenfeld, J. \& Papautsky, I. Reconfigurable virtual electrowetting channels. Lab. Chip 12, 758 (2012).

10. Choi, K., Ng, A. H. C., Fobel, R. \& Wheeler, A. R. Digital Microfluidics. Annu. Rev. Anal. Chem. 5, 413-440 (2012).

11. Lee, W. C., Heo, Y. J. \& Takeuchi, S. Wall-less liquid pathways formed with three-dimensional microring arrays. Appl. Phys. Lett. 101, 114108 (2012).

12. Walsh, E. J. et al. Microfluidics with fluid walls. Nat. Commun. 8, 816 (2017). 
13. Keerthi, A. et al. Ballistic molecular transport through two-dimensional channels. Nature 558, $420-424(2018)$.

14. Shang, L., Cheng, Y. \& Zhao, Y. Emerging Droplet Microfluidics. Chem. Rev. 117, 7964-8040 (2017).

15. Zhao, W., Cheng, R., Miller, J. R. \& Mao, L. Label-Free Microfluidic Manipulation of Particles and Cells in Magnetic Liquids. Adv. Funct. Mater. 26, 3916-3932 (2016).

16. Coey, J. M. D., Aogaki, R., Byrne, F. \& Stamenov, P. Magnetic stabilization and vorticity in submillimeter paramagnetic liquid tubes. Proc Natl Acad Sci 106, 8811-8817 (2009).

17. Caravan, P., Ellison, J. J., McMurry, T. J. \& Lauffer, R. B. Gadolinium(III) Chelates as MRI Contrast Agents: Structure, Dynamics, and Applications. Chem. Rev. 99, 2293-2352 (1999).

18. Ferrand, J., Favreau, L., Joubaud, S. \& Freyssingeas, E. Wetting Effect on Torricelli's Law. Phys. Rev. Lett. 117, 248002 (2016).

19. Rosensweig, R. E. Ferrohydrodynamics. (Dover Publications, 2014).

20. Posocco, P. et al. Interfacial tension of oil/water emulsions with mixed non-ionic surfactants: comparison between experiments and molecular simulations. RSC Adv 6, 4723-4729 (2016).

21. Owusu Apenten, R. K. \& Zhu, Q.-H. Interfacial parameters for selected Spans and Tweens at the hydrocarbon-water interface. Food Hydrocoll. 10, 27-30 (1996).

22. Byrnes, J. et al. Hemolysis During Cardiac Extracorporeal Membrane Oxygenation: A CaseControl Comparison of Roller Pumps and Centrifugal Pumps in a Pediatric Population. ASAIO J. 57, $456(2011)$. 
23. Omar, H. R. et al. Plasma Free Hemoglobin Is an Independent Predictor of Mortality among Patients on Extracorporeal Membrane Oxygenation Support. PLOS ONE 10, e0124034 (2015).

24. Dalton, H. J. et al. Factors Associated with Bleeding and Thrombosis in Children Receiving Extracorporeal Membrane Oxygenation. Am. J. Respir. Crit. Care Med. 196, 762-771 (2017).

25. Valladolid, C., Yee, A. \& Cruz, M. A. von Willebrand Factor, Free Hemoglobin and Thrombosis in ECMO. Front. Med. 5, (2018).

26. Wilson, A. M. M. M. et al. Hemolysis risk after packed red blood cells transfusion with infusion pumps. Rev. Lat. Am. Enfermagem 26, (2018).

27. Prahl, S. Optical absorption of hemoglobin. https://omlc.org/spectra/hemoglobin/index.html (1999).

28. Baskin, L., Dias, V., Chin, A., Abdullah, A. \& Naugler, C. Chapter 3 - Effect of Patient Preparation, Specimen Collection, Anticoagulants, and Preservatives on Laboratory Test Results. in Accurate Results in the Clinical Laboratory (eds. Dasgupta, A. \& Sepulveda, J. L.) 19-34 (Elsevier, 2013).

29. Jaouen, P., Vandanjon, L. \& Quéméneur, F. The shear stress of microalgal cell suspensions (Tetraselmis suecica) in tangential flow filtration systems: the role of pumps. Bioresour. Technol. 68, 149-154 (1999).

30. Kamaraju, H., Wetzel, K. \& Kelly, W. J. Modeling shear-induced CHO cell damage in a rotary positive displacement pump. Biotechnol. Prog. 26, 1606-1615 (2010).

31. Vázquez-Rey, M. \& Lang, D. A. Aggregates in monoclonal antibody manufacturing processes. Biotechnol. Bioeng. 108, 1494-1508 (2011). 
32. Wang, S. et al. Shear contributions to cell culture performance and product recovery in ATF and TFF perfusion systems. J. Biotechnol. 246, 52-60 (2017).

33. Nesta, D. et al. Aggregation from Shear Stress and Surface Interaction: Molecule-Specific or Universal Phenomenon? BioProcess International 30, 30-39 (2017).

34. Hejazian, M., Li, W. \& Nguyen, N.-T. Lab on a chip for continuous-flow magnetic cell separation. Lab. Chip 15, 959-970 (2015).

35. Eijkel, J. C. T. \& Berg, A. van den. Nanofluidics: what is it and what can we expect from it? Microfluid. Nanofluidics 1, 249-267 (2005).

36. Bocquet, L. \& Charlaix, E. Nanofluidics, from bulk to interfaces. Chem Soc Rev 39, 1073-1095 (2010).

37. Bocquet, L. \& Tabeling, P. Physics and technological aspects of nanofluidics. Lab. Chip 14, 3143-3158 (2014).

Supplementary Information See published version

Data and Code Availability Source data for figure(s) 2, 3, 4 (and extended data figures containing data graphs) are provided with the paper. Any other data that support the findings of this study, and the python code for calculating magnetic fields, are available on the Zenodo data repository, doi: $10.5281 /$ zenodo. 3603029

Acknowledgments: We acknowledge the support of the University of Strasbourg Institute for Advanced Studies (USIAS) Fellowship, The 'Chaire Gutenberg' of the Région Alsace (J.M.D.C.), French National Research Agency (ANR) through the Programme d'Investissement d'Avenir under contract ANR-11-LABX-0058_NIE within the Investissement d'Avenir program ANR-10-IDEX0002-02, and SATT Conectus funding. This project has received funding from the European Union's 
Horizon 2020 research and innovation programme under the Marie Skłodowska-Curie grant agreement No 766007. We acknowledge the Paul Scherrer Institut, Villigen, Switzerland for provision of synchrotron radiation beamtime at beamline TOMCAT of the SLS. We are grateful to Dr. Hu Boping, of San Huan Corporation for giving us thin magnetic bilayer sheets. We thank Fabien Chevrier for technical support, and the staff of the STnano nanofabrication facility for help in sample fabrication. We thank Nina Matoussevitch for the synthesis of ferrofluids. We would like to thank Flor Sacarelli and Georges Formon for additional AAS measurements, Andrejs Cebers, University of Latvia Riga for the use of ANSYS 18, and S. Pottier for advice in the project.

Author contributions: J.M.D.C., B. D. and T.M.H. conceived and initiated the project; T.A. and P. D. performed most of the experiments and modelling; T.A., J.M.D.C., B.D., P. D. and T.M.H. designed the microfluidics setups; A.A.D. performed slip length experiments and modelling; P.D. carried out the magnetic modelling; A.S. synthesized Magoil; L.G. characterized liquid and pumping properties; A.B. led the X-Ray tomography experiments; C.B., T.A, T.M.H, and P.M. designed and conducted the blood experiments; T.A., J.M.D.C., B.D., P.D. and T.M.H. drafted the manuscript, and all authors discussed and contributed to the paper in its final form.

Competing interests: T.M.H. holds shares in Qfluidics, a company devoted to the commercialization of the liquid tube technology presented in this work. P.D., B.D., J.M.D.C. and T.M.H. are co-inventors on patents protecting the technology (WO2018134360A1, pending) for which all parts of the manuscripts are covered. 


\section{Methods}

\section{M1 Synthesis of Rare Earth Oil}

Inspired by the well-known gadolinium complexes of DTPA (diethylenetriaminepentacetate), used as paramagnetic contrast agents in magnetic resonance imaging (MRI), we prepared an amphiphilic complex of DTPA with the paramagnetic rare-earth ion holmium (III), bearing two hydrophobic side chains connected to the DTPA moiety through ester linkage. In particular, by reaction of DTPA dianhydride with the branched alcohol 2-butyl-1-octanol we obtained a tricarboxylic chelating ligand, which upon complexation with $\mathrm{Ho}^{3+}$ under alkaline conditions afforded a stable neutral HoDTPA complex. The latter, insoluble in water, was mixed with 2-butyl-1-octanol (30\% wt of the latter) affording a water-immiscible homogeneous fluid (Magoil) featuring positive magnetic susceptibility. The magnetic properties of the neutral Ho-DTPA complex (i.e. before mixing with the alcohol) were evaluated by NMR measurement, using the Evans method ${ }^{38}$, giving a value $4.40 \times 10^{-7} \mathrm{~m}^{3} \mathrm{~mol}^{-1}$ for the molar susceptibility, which is comparable with that measured for the inorganic salt $\mathrm{Ho}(\mathrm{AcO})_{3}$, as well as of the order of magnitude expected for $\mathrm{Ho}^{3+} \cdot{ }^{39}$ Our paramagnetic oil is a transparent non-ionic liquid, slightly pink-yellowish because of the fluorescence of $\mathrm{Ho}(\mathrm{III})$, and it is physico-chemically stable in contact with aqueous solutions for several hours. Over longer periods of contact, hydrolysis occurs. Overall these characteristics make it different from other magnetic fluids, such as: ferrofluids (i.e. black colloidal suspensions of nanometre-sized magnetic particles), or magnetic room temperature ionicliquids (RTIL). Lastly, we stress that the preparation of the oil could be easily scaled up to $25 \mathrm{~g}$, and that we used also different trivalent rare-earth ions, such as erbium and terbium, obtaining analogous results.

\section{M2 Preparation of the Ho-DTPA-(2-buthyl-1-octanol)2 based paramagnetic oil}

DTPA dianhydride (5 g, $14 \mathrm{mmol})$ was dispersed in dry dimethylformamide (100 mL) under argon atmosphere, and the obtained suspension was heated to $70-75^{\circ} \mathrm{C}$ under stirring, until complete solubilization was observed. After that, 2-butyl-1-octanol (5.5 g, $28 \mathrm{mmol}, 2$ eq.), dissolved in $10 \mathrm{~mL}$ DMF, was added dropwise to the above solution and the resulting homogeneous mixture was left under 
stirring at $50^{\circ} \mathrm{C}$ for $4 \mathrm{~h}$. The completeness of the reaction was monitored by liquid chromatography/mass-spectrometry (LC-MS). Afterwards, the solvent was removed (rotary evaporation + high vacuum pump) to give $\mathbf{2}$ as a sticky yellowish solid that was used without further purification (Fig. E2a).

To a stirred $0.2 \mathrm{M}$ solution of the ligand 2 in ethanol $(\mathrm{EtOH})$, an aqueous $1 \mathrm{M}$ solution of $\mathrm{NaOH}$ ( 3 eq.) was added dropwise at room temperature, followed by the addition of $\mathrm{HoCl}_{3} .\left(6 \mathrm{H}_{2} \mathrm{O}\right)(1.5$ eq. with respect to 2), dissolved in the proper amount of water so to have a final $0.1 \mathrm{M}$ solution of the complex of $\mathbf{3}$ in ethanol/water 1:1. The resultant homogeneous mixture was left stirring at room temperature until a viscous pink oil, that responds to magnetic fields, separated out from the water phase (typically $0.5-1 \mathrm{~h}$ ). After that, the solution was concentrated by rotary evaporation to remove EtOH, and the water phase extracted with dichloromethane. The organic phase was separated, dried over $\mathrm{MgSO}_{4}$ and rotary evaporated to give the complex $\mathbf{3}$ as a glassy solid. Finally, the latter was dissolved in the minimum amount of dichloromethane, and the fatty alcohol 2-butyl-1-octanol (30 wt $\%)$ was added to the resultant solution. Afterwards, the mixture was rotary evaporated under high vacuum to completely eliminate dichloromethane, resulting in the target homogeneous transparent paramagnetic oil. The absorbance of the paramagnetic oil (Fig. E2b) matches the absorption peaks of $\mathrm{Ho}^{3+} .^{40}$

\section{M3 Measurement of the magnetic susceptibility of the paramagnetic oil}

The magnetic susceptibility of the neutral complexes $\mathbf{3}$ was measured by the Evans method, using NMR spectroscopy ${ }^{38}$. This method relies on the fact that the chemical shift of the ${ }^{1} \mathrm{H}$ NMR signals of a molecule depend on the bulk susceptibility of the medium. In particular, we used the resonance line of the residual proton of $\mathrm{CDCl}_{3}$ (i.e. the NMR solvent) as a reference, by comparing its chemical shift in pure $\mathrm{CDCl}_{3}$ and in a $\mathrm{CDCl}_{3}$ solution containing the complex 3 . From the difference in the chemical shifts we could calculate the mass and molar susceptibilities of $\mathbf{3}\left(\chi_{\text {mass }}=4.88 \times 10^{-7} \mathrm{~m}^{3} \mathrm{~kg}^{-1} ; \chi_{\text {mol }}=4.40\right.$ $\left.\times 10^{-7} \mathrm{~m}^{3} \mathrm{~mol}^{-1}\right)$. 


\section{M4 Magnetic Properties of the Ferrofluids}

Magnetization measurements were performed using vibrating sample magnetometer with a compact variable permanent magnet source ${ }^{41}$. Samples were mounted into 3D printed sample holders with cylindrical chambers of diameter $3 \mathrm{~mm}$ and length 3.88 or $5.88 \mathrm{~mm}$, ensuring a well-defined demagnetization factor, and maximal use of the uniform region of the pick-up coils.

The magnetic field dependence of the magnetization was assumed to be due to dispersed non-interacting spherical particles in a non-magnetic medium, and the data was fitted with either a single component, or two component Langevin function. After correcting for non-zero magnetic field offsets, the applied field $H$ was corrected for the appropriate demagnetization factor, $N^{42}$ to get the internal field $H^{\prime}$. Additional magnetization measurements were performed by measuring the inductance of solenoids immersed in the ferrofluids. The solenoid inductance was measured using a Hameg 8118 LCR bridge meter at $1 \mathrm{kHz}$. See SI section I for Langevin and demagnetisation factor equations.

\section{M5 Remnant Magnetization of Permanent Magnets}

The remnant magnetization $M_{r}$ of the permanent magnets used was measured by profiling the external field with a Gauss-meter in the $z$ direction along the central axis and fitting the resultant profile to analytical expressions ${ }^{43}$ (see SI section II for equations).

\section{M6 Magnetic Field Simulations}

Assuming magnetically transparent, uniformly magnetised permanent magnets, three-dimensional magnetic fields can be calculated with analytical expressions based on the surface charge model. ${ }^{44,45}$ The magnetic field at each point is summed over the contributions of each magnet in an assembly. Full expressions are presented in SI section III.

\section{M7 Surface Tension of Magnetic Liquids}

A simple home-build pendent drop setup was used to measure the surface tension of the magnetic liquids. The solutions were gradually dispensed in a stepwise fashion through a polished $74 \mu \mathrm{m}$ diameter glass capillary using a syringe pump into a glass cuvette in a 3D printed support. The pendent drops were imaged using a Canon EOS 50D and Tamron superzoom 18-270 mm lens in RAW mode. RAW 
images were converted to monochrome TIFF files, with image processing being carried out in Image $\mathrm{J}^{46}$, and the surface tension calculated using "Pendent_Drop", an ImageJ plugin ${ }^{47}$.

The drops were gradually enlarged, with multiple images taken at each size until the drops broke off, and this was repeated at least 6 times. The values reported in Table E1 are pooled mean and variance, calculated from at least 6 different droplets, for at least 15 droplet sizes, and 3 images per size. Overall, between 30 - 100 images of droplets of various sizes were used per surface tension value. Surface tension measurements were cross-checked with a Kruss Drop Shap Analyser DSA100 and found to be in agreement.

\section{M8 Method for Self-Consistent Diameter Calculation using the Full Model (FM)}

For a given set of conditions, (i.e. permanent magnet remnant magnetization, quadrupole spacing, ferrofluid nanoparticle diameter and volume fraction, surface tension), $d$ can be calculated in a selfconsistent manner by calculating the magnetic field and magnetization at a position $d / 2$ using the scheme of M5, next generating a new $d$ using Eq. (3), use this as a new input $d$, and looping repeatedly until the difference between input and output is negligible.

\section{M9 Side-View Optical Imaging and Pressure/Flow Measurements}

Images were acquired with either i) a Leica MZ16 stereo microscope with a Leica IC80HD digital camera, or ii) a Nikon SMZ745T stereo microscope and a SONY 1/1.8” 20im/sec 1600x1200 pix color CCD camera, while an Elveflow OB1 pressure driven pump and Elveflow FS4 flow sensors were used to set the applied pressure and measure the resulting flow rates in and out of the antitubes. All image processing and analysis was carried out in Image ${ }^{46}$. Image thresholding was performed using Otsu's clustering method, with the resulting image converted to a binary mask. Particle analysis and circle fitting was performed on each image, resulting in cross-sectional area, and roundness/circularity. Example cross-sections are shown in Fig. E4d.

\section{M10 Optical Transmission Imaging}

A Zeiss Axiovert 200M inverted microscope with a 5x NA=0.15 objective and Andor Zyla 4.2 SCMOS detector were used to image through 3D printed microfluidic devices. These devices consist 
of a $200 \mu \mathrm{m}$ high and $1 \mathrm{~mm}$ wide main channel, a right-angled Y-junction for 2 water inlets, a ferrofluid inlet which joins the channel at its centre, a single outlet, and space to place 4 magnets to generate a quadrupolar field centred in the main channel. The device is sealed with a thin glass coverslip.

For stabilising antitubes, two methods were used, i) the channel was prefilled with water with the ferrofluid injected, gradually displacing the water inside the channel, or ii) the channel was prefilled with ferrofluid, with water injected to displace ferrofluid.

Tube diameter was estimated using ImageJ. Plot profiles were generated by taking column averages of images (Fig. E3 f,g), followed by subtraction of the background, leading to a sharp peak due to the lower absorption of water. This is fitted with a Gaussian peak function, whose full width half max corresponded to tube diameters measured from the optical side view technique.

\section{M11 Top-View Optical Imaging Through Magoil}

A Nikon SMZ745T stereo microscope and a SONY 1/1.8" 20im/sec 1600x1200 pixel colour CCD camera were used to image water tubes inside Magoil. Similar to the X-ray, and optical, transmission images, the optical images were rotated, inverted (Fig. E8), and profiles of column averages were extracted for data fitting. The background was subtracted, and the resulting peaks fitted with a gaussian function.

\section{M12 X-Ray Transmission Imaging}

A MyRay dental X-ray imaging system was adapted for imaging through ferrofluids, consisting of an X-ray source (MyRay RX-DC eXtend) and a detector (MyRay Zen X T1) were purchased from Castebel (Waver, Belgium), and confined inside an in-house constructed box covered by lead plates. In general, X-ray emission at $65 \mathrm{kV}$ and $6 \mathrm{~mA}$ with the exposure time of $0.1 \mathrm{~s}$ was used to obtain a good contrast image of water antitubes confined in ferrofluids.

To measure the tube diameter, first a background measurement with a microfluidic device (Fig. E3 a,b) fully filled with ferrofluid was taken. The image processing loop (also in ImageJ) consisted of taking the average of 10 images, rotated to have the tube fully vertical, with the intensity values inverted (Fig. E3 c). Next a plot profile is generated by taking column averages (Fig. E3 d, red line). Then a 
freshly cleaned device is filled with water, and progressively ferrofluid was added in $50 \mu 1$ steps at a flow rate of $100 \mu 1 / \mathrm{min}$, with 10 images taken at each step. The same imaging process carried out as with the background, followed by subtraction of the background plot profile, leading to a sharp peak due to the lower absorption of water (Fig. E3 e). This is fitted with a Gaussian peak function, whose full width half max corresponded to tube diameters measured from the optical side view technique. The procedure of adding ferrofluid was continued until a continuous tube is no longer observed. For improved contrast sensitivity, additional transmission images were taken with a Hamamatsu C7942CK12 flat panel sensor in place of the MyRay Zen X T1, as shown in Fig. E1 a,b.

\section{M13 Synchrotron X-Ray Tomography}

Phase contrast synchrotron X-ray tomography was carried out at the X02DA TOMCAT beamline of the Swiss Light Source (SLS) at the Paul Scherrer Institute (Villigen, Switzerland). The X-ray beam, produced by a $2.9 \mathrm{~T}$ bending magnet on a $2.4 \mathrm{GeV}$ storage ring (with ring current $I=400 \mathrm{~mA}$ ), was monochromated to $30 \mathrm{keV}$. We used a high-speed CMOS detector (PCO.Edge 5.5) coupled to a KinoOptik 1x visible light objective with a $300 \mu \mathrm{m}$-thick LuAG:Ce scintillator, for a $16.6 \mathrm{~mm} \times 14.0$ $\mathrm{mm}$ field of view, and $6.5 \mu \mathrm{m}$ effective pixel size. The sample-to-detector distance was $327 \mathrm{~mm}$. Raw images were acquired with 50 ms exposure times across 1500 tomographic projections. 20 dark and 100 flat field images were collected for each scan.

The samples consisted of 3D printed supports with circular cross-section and a $1.5 \mathrm{~mm}$ diameter channel. There is a groove on the top and bottom of the holder to accommodate two 10x10x10 mm N42 grade $\mathrm{Nd}_{2} \mathrm{Fe}_{14} \mathrm{~B}$ magnets on each side at a distance of $3 \mathrm{~mm}$. The $3 \mathrm{D}$ print is designed so that the centre of the null field is close to the centre of the fluidic channel. The channels were prefilled with water containing 1 vol.\% Tween-20 surfactant and imaged after sequential injection of known volumes of a ferrofluid using a syringe pump.

Prior to further analysis, each projection was corrected with the respective dark and flat-field image. Computed tomography (CT) reconstruction was performed using the gridrec algorithm ${ }^{48}$, enabling fast reconstructions of large data sets. Tomographic slices were rendered using ImageJ 1.52i, and the volumetric cross-sections were rendered using Paraview 5.6.0. 


\section{M14 Antitube Durability}

The durability of antitube was tested by varying the flow rate of water with a syringe pump to determine the threshold value when ferrofluid is sheared out. For this test, a fluidic chip which consists of four $9 \times 9 \times 50 \mathrm{~mm}$ magnets with the gap of $9 \mathrm{~mm}$ between magnets was used. No major leakage of ferrofluid was observed for all the measurement, and the threshold value was reported for the flow rate when iridescence due to a thin film of oil on the water surface was observed. The observed data (Fig. E4 a,b) is valid only for this specific magnet arrangement and fluidic circuit, as the magnetic field distribution varies when the size and arrangement of magnets are changed, and outlets perpendicular to the main channel inhibit further leakage. In general, the threshold value increases when more viscous or magnetically stronger ferrofluid are used for antitube formation and the threshold value decreases as the antitube diameter decreases.

\section{M15 Quantitative analysis of water contamination level by magnetic media}

The amount of magnetic media leaked into water was quantified by atomic absorption spectroscopy (AAS, Agilent Technologies 200 Series AA). The emission wavelength of iron (372 nm) and holmium $(559 \mathrm{~nm})$ was used for ferrofluids and Magoil, respectively. The standard samples for iron were prepared by dissolving iron metal in hydrochloric acid and diluted by distilled water to obtain a series of concentrations to construct a calibration curve. Each point is from five consecutive measurements. $1 \mathrm{~mL}$ of ferrofluid (MKC and MD4) and $25 \mathrm{~mL}$ of distilled water were mixed in a separatory funnel and left overnight. $20 \mathrm{~mL}$ of the distilled water from the funnel was transferred to a vial and water was removed by using a rotary evaporator. $1 \mathrm{~mL}$ of hydrochloric acid was added to the vial to dissolve any remaining iron. The iron solution was diluted to make total $50 \mathrm{~mL}$ and used for AAS. Similarly, $1 \mathrm{~mL}$ of Magoil was mixed with $25 \mathrm{~mL}$ of distilled water. Five consecutive measurements were performed for each sample to build the statistics. The emission intensity of 0.049 \pm 0.002 and $0.050 \pm 0.004$ was observed from the water sample that was in contact with MKC and MD4, respectively. Using the calibration curve and error propagation analysis, the concentration of the iron from water in contact with MKC and MD4 was determined as $0.78 \pm 0.05 \mathrm{ppm}$ and $0.79 \pm 0.08$ ppm. 
Additional checks were made by flowing water through three different antitube diameters, 3.5 $\mathrm{mm}, 1.5 \mathrm{~mm}$ and $0.8 \mathrm{~mm}$, respectively. The cavity containing both ferrofluid and water is $\mathrm{xx} \mathrm{mm}$ in diameter. We used DI water as transported fluid and the surrounding ferrofluid was APG1141 with viscosity 5 Pa.s. Three flowrates were used corresponding to $10 \%, 50 \%$ and $75-80 \%$ of the threshold flowrate for each diameter $\left(Q_{\text {thres }}=80,60\right.$, and $30 \mu \mathrm{l} / \mathrm{min}$ respectively, cf. Fig. E4a,b). The setup consists of an OB1 pressure driven pump (Elvesys) connected to a water reservoir which is then connected to the antitube housed in a 3D printed support. At the outlet the flow is split into two streams. One passes directly to a falcon tube and the other flows adjacent to a $6 \times 6 \times 50 \mathrm{~mm}$ magnet before entering a second falcon tube. This additional magnet was placed with its long axis parallel to the channel flow, and is there to capture any ferrofluid that may inadvertently shear out under flow. The water was flown continuously for 1.5 hours, and $45 \mathrm{ml}$ samples were collected in each falcon tube at the beginning, middle, and end of the experiment. All solutions were evaporated, followed by the addition of $1 \mathrm{~mL}$ concentrated $\mathrm{HCl}$, which was allowed to react for $1 \mathrm{~h}$ and diluted with $45 \mathrm{~mL}$ of ultrapure $\mathrm{H}_{2} \mathrm{O}$. The entire procedure was repeated in order to produce a total of 6 samples per experimental condition, with the data reported in Fig. E4f being the average values, and error bars being the standard deviations. Here we found that all Fe concentrations detected were below $0.3 \mathrm{ppm}$, with the majority of them within one standard deviation for a series of blank measurements.

Magoil hydrolyses in a few hours, and therefore $20 \mathrm{~mL}$ of water was transferred to a vial after one hour and diluted to make total $50 \mathrm{~mL}$ of the sample for AAS. Holmium (III) chloride was dissolved in distilled water and diluted to obtain a calibration curve. The emission intensity of $0.058 \pm 0.001$ was observed from the water sample that was in contact with Magoil, which corresponds to $2.6 \pm 0.1 \mathrm{ppm}$ based on the calibration curve. 


\section{M16 Construction of Qpump}

The Qpump housing was 3D printed using F170 (Stratasys Ltd.) with ABS (Acrylonitrile Butadiene Styrene)-M30. $\mathrm{Nd}_{2} \mathrm{Fe}_{14} \mathrm{~B}$ arc magnets were purchased from NEOTEXX (TR-036.5-33-20NN and -SN, TR-028.5-25-20-NN and -SN). A high-power gear motor (RobotShop Inc., Devantech $24 \mathrm{~V}$ ) was used to rotate the inner rotor of the Qpump. The fluidic chips for were 3D printed using a Form 2 print (Formlabs Inc.) with a clear resin (FLGPCL02), which were then connected to Tygon tubing (Saint-Gobain ${ }^{\mathrm{TM}}$, ND 100-65, 1/16"ID x 1/8"OD).

\section{M17 Blood Pumping and Analysis}

$50 \mathrm{~mL}$ of human blood donated from a unique donor at l'Établissement français du sang (EFS) Strasbourg was used for each set of experiments. The blood was collected on anticoagulant with hirudin (100 units $/ \mathrm{mL}$ ). Six sets of $6 \mathrm{~mL}$ tubes were prepared: as a negative control (not used in a pump), three for use with a Qpump, and two for a peristaltic pump as one set of experiment. To build statistics, blood from three different donors was used to construct the data reported here. The rotation rate of motors was adjusted to obtain the flow rate of each pump at $\sim 1.5 \mathrm{ml} / \mathrm{min}$. Tygon tube (Saint-Gobain ${ }^{\mathrm{TM}}$, Tygon 3350, 1/16"ID x 3/16"OD) with stoppers was used with the peristaltic pump (Fischer Scientific, СТP300).

After one hour of blood pumping in a closed circuit (main text Fig. 4c), the whole blood from each tube was analysed using a haematology analyser (Sysmex Europe GmbH, XN-1000). Then, the whole blood was centrifuged (SORVALL RC3BP, $10 \mathrm{~min}$ at $250 \mathrm{~g}$, room temperature) to obtain platelet rich plasma (PRP) for UV-vis spectroscopy (Agilent Technologies, Inc., Cary 8454) and light transmission aggregometry (APACT 4004). For UV-vis spectroscopy, PRP was 25 times diluted with phosphate buffer. Light transmission was recorded during 5 min while platelets aggregated after the addition of $30 \mu \mathrm{L}$ of agonist (thrombin receptor-activating peptides: TRAP $10 \mu \mathrm{M}$ ) into $270 \mu 1$ of PRP.

To perform electron microscopy imaging of platelets, samples were prepared as follows: first $100 \mu 1$ of PRP was added to $400 \mu 1$ glutaraldehyde $2.5 \%$ (euromedex, 16210) (in cacodylate, CACO, buffer) in a $15 \mathrm{ml}$ tube and incubated overnight at $4^{\circ} \mathrm{C}$. Then the solution was centrifuged for $8 \mathrm{~min}$ at $2000 \mathrm{~g}$ to obtain pellet platelets which were then resuspended with $2 \mathrm{ml} \mathrm{CACO}$ buffer for washing. This 
solution was centrifuged again for $8 \mathrm{~min}$ at $2000 \mathrm{~g}$ and the collected platelets were resuspend with 250 $\mu 1$ of CACO buffer. Finally, the solution was diluted 10 times and $100 \mu 1$ of diluted solution was deposited on a poly-L-lysine (Sigma Aldrich Chimie, P8920) coverslip (Euromedex, 72226-01) to sediment the platelets at room temperature. After $30 \mathrm{~min}$, the excess solution was removed, and the substrate was washed twice with CACO buffer for 5 minutes. Dehydration of the substrate was done with ethanol (LPCR: Les Produits Chimiques de la Robertsau, 20821296): 70\% (3 x 5 min), 85\% (1 x $5 \mathrm{~min}), 95 \%(1 \times 5 \mathrm{~min})$, and $100 \%(2 \times 30 \mathrm{~min})$. The substrate was then desiccated with hexamethyldisilazane (LPCR, 112186.0100): 1/4 HMDS + 3/4 ethanol 100\% (1 x 5 min), 1/2 HMDS $+1 / 2$ ethanol 100\% ( 1 x 5 min), $3 / 4$ HMDS + 1/4 ethanol 100\% ( $1 \times 5$ min), and pure HMDS ( 2 x 5 min). The coverslip was fixed on an aluminium support mount (Euromedex, 75230) with conductive carbon cement (Euromedex, 12664). The sample was metallized (coating by $\sim 10 \mathrm{~nm}$ layer of platinum and palladium) using Cressington 208 HR Sputter coater and observed with Phenom Pro Desktop SEM for high-quality imaging.

Ferrofluid contamination in the pumped blood was determined using the protocol of M15, along with a complementary method of measuring the magnetic moment of the whole blood samples in a SQUID magnetometer (Quantum Design MPMS3 SQUID-VSM). First, the ferrofluid MKC was dried by rotary evaporation to measure the magnetic response without the hydrocarbon carrier fluid. Similar to the Langevin function fitting of method M4, the concentration of superparamagnetic magnetite nanoparticles in the powder was found to be $76 \%$.

Next, 8 whole blood samples, 4 pumped through two different Qpumps, 2 pumped through a peristaltic pump, and 2 controls (not pumped at all), were lyophilised before mounting in gelatine capsules. The control and peristaltic pumped samples showed only a diamagnetic susceptibility at room temperature, as expected for oxyhaemoglobin, with an average susceptibility of $-2.3 \pm 0.3 \mathrm{~A} \mathrm{~m}^{2} \mathrm{~kg}^{-1} \mathrm{~T}^{-}$ ${ }^{1}$ (after correction for sample holder contributions). In these blood samples deoxyhaemoglobin is only visible at low temperatures, where a Brillouin function fit reveals an average of 4 unpaired spins, as expected. In contrast, the 4 magnetically pumped samples returned an average superparamagnetic 
moment of $26.2 \pm 14.8 \times 10^{-3} \mathrm{~A} \mathrm{~m}^{2} \mathrm{~kg}^{-1}$, which is the equivalent of $285 \pm 161 \mathrm{ppm}$ of $\mathrm{Fe}_{3} \mathrm{O}_{4}$ per gram of blood.

M18 Derivation of Equations (1) and (2) Effective Slip Length of a Flow in a Cylindrical Ferrofluid

We use the steady state Stokes equations in radial coordinate for ferrofluid as well as honey. The fluid landscape is divided in three regions namely the honey flow (region I), ferrofluid with shear flow (region II) and reverse flow of ferrofluid (region III) (see Fig. E5e). The solid walls are given zero velocity boundary condition, and the interface between the liquids has continuous velocity and shear stress. Since there is no net flow of ferrofluid, the flow rate of ferrofluid in region III is equal and opposite in region II. This can be seen in the normalised velocity profile in Fig. E4e which was also confirmed by numerically solving the Navier-Stokes equation in Fig. E5a-d. Finally, solving the Stokes equations with the respective boundary conditions, the effective slip length is defined in terms of the flow rate (for a fixed pressure gradient) in the system. The full derivation is presented in SI section III.

\section{M19 Derivation of Equilibrium Diameter Equation (3)}

The approach starts with the magnetically augmented Bernoulli equation, choosing appropriate boundary conditions at two points (see Fig. E5f), one at the centre of the antitube in the null field region, $\mathrm{P}_{1}$, and the other at the boundary between the inner solution and the magnetic liquid, $\mathrm{P}_{2}$, and solving the Bernoulli equation to arrive at an expression due to the balance of energies at the interface. A derivation is provided in SI section IV.

\section{M20 Derivation of Linear (LM) and Saturation Models (SM) for the Equilibrium Tube Diameter}

Equation (3) is simplified to equation (4) by linearising the magnetic field dependence inside the quadrupole, assuming a linear magnetic susceptibility for the ferrofluid, and inserting both results into equation 3. A full derivation is described in SI section V.

\section{M21 Computational Fluid Dynamics}

Finite element methods using ANSYS 18 were used to simulate fluid dynamics of a honey antitube inside EMG900 ferrofluid. A full 3-dimensional simulation was performed, with the following 
boundary conditions applied: at the inlet we define the flow rate $(Q=175 \mu 1 / \mathrm{min})$, and the outlet is set to atmospheric pressure. The interface between liquids is defined as two walls with constant magnitude of shear stress $(\tau)$. We vary the shear stress until the velocity at the interface becomes continuous. Once the shear stress and velocity becomes continuous the solution is accepted. Here $\tau=0.45 \mathrm{~Pa}$ for the semiinfinite case and $\tau=0.47 \mathrm{~Pa}$ for the case where we include the effect of curved inlet and outlet. The reverse flow as predicted by the analytical model is clearly seen in the simulations. The antitube diameter is $1.2 \mathrm{~mm}$ and the cavity size is $4.4 \mathrm{~mm}$. The fluids used are honey with viscosity $10 \mathrm{~Pa} . \mathrm{s}$ and EMG900 (ferrofluid) with viscosity 0.06 Pa.s. Note that to simplify the simulations we use free slip conditions at the curved inlet and outlet region. This is because the change in the velocity in the curved inlet and outlet region is dominated solely by the change in diameter.

When comparing an infinite channel (Fig. E5 a,c) to a finite channel (Fig. E5 b,d) we observe the same counterflow of ferrofluid. For the finite channel, the velocity away from either the inlet or outlet has a flat, plug-like profile (Fig. E5d) identical to the infinite channel (Fig. E5b), but at the outlet there is a large drop in the flow velocity as the volumetric flow rate is fixed and the channel widens. Furthermore the majority of the pressure drop occurs at both the inlet and outlet where the channel diameter changes (Fig. E5c). Additionally, we observe the same pressure drop inside the finite antitube away from the inlet and outlet, as found for the infinite antitube (Fig. E5a).

\section{Additional References}

38. Evans, D. F. The determination of the paramagnetic susceptibility of substances in solution by nuclear magnetic resonance. J. Chem. Soc. 2003-2005 (1959).

39. Coey, J. M. D. Magnetism and Magnetic Materials. (Cambridge University Press, 2010).

40. Sastri, V. R., Perumareddi, J. R., Rao, V. R., Rayudu, G. V. S. \& Bünzli, J.-C. G. Modern Aspects of Rare Earths and their Complexes. (Elsevier, 2003). 
41. Cugat, O., Byrne, R., McCaulay, J. \& Coey, J. M. D. A compact vibrating-sample magnetometer with variable permanent magnet flux source. Rev. Sci. Instrum. 65, 3570-3573 (1994).

42. Wysin, G. M. Demagnetization fields. (Kansas State University, 2012). https://www.phys.kstate.edu/personal/wysin/notes/demag.pdf

43. Furlani, E. P. Permanent Magnet and Electromechanical Devices. (Academic Press, 2001).

44. Yang, Z. J., Johansen, T. H., Bratsberg, H., Helgesen, G. \& Skjeltorp, A. T. Potential and force between a magnet and a bulk $\mathrm{Y}_{1} \mathrm{Ba}_{2} \mathrm{Cu}_{3} \mathrm{O}_{7} \delta$ superconductor studied by a mechanical pendulum. Supercond. Sci. Technol. 3, 591 (1990).

45. Camacho, J. M. \& Sosa, V. Alternative method to calculate the magnetic field of permanent magnets with azimuthal symmetry. Rev. Mex. Física E 59, 8-17 (2013).

46. Schindelin, J. et al. Fiji: an open-source platform for biological-image analysis. Nat. Methods $\mathbf{9}$, $676-682(2012)$.

47. Daerr, A. \& Mogne, A. Pendent_Drop: An ImageJ Plugin to Measure the Surface Tension from an Image of a Pendent Drop. J. Open Res. Softw. 4, e3 (2016).

48. Marone, F. \& Stampanoni, M. Regridding reconstruction algorithm for real-time tomographic imaging. J. Synchrotron Radiat. 19, 1029-1037 (2012). 


\section{Extended Data}

\section{Extended Data Table E1}

Measured physical properties of the two commercial ferrofluids (Ferrotec Corporation,

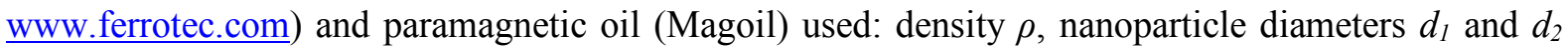
(for bimodal size distributions), volume fractions $\phi_{1}$ and $\phi_{2}$ of magnetite in the suspension (corresponding to particles of size $d_{1}$ and $d_{2}$ ), initial susceptibility $\chi$, surface tension $\sigma$ with air, surface tension with water, and surface tension with $1 \%$ tween-20 in water.

\begin{tabular}{lccccccccc}
\hline Sample & $\rho$ & $\mathrm{d}_{1}$ & $\mathrm{~d}_{2}$ & $\phi_{1}$ & $\phi_{2}$ & $\chi$ & $\sigma_{\text {air }}$ & $\begin{array}{c}\sigma_{\text {water }} \\
\mathrm{mN} \mathrm{m}^{-1}\end{array}$ & $\begin{array}{c}\sigma_{\text {tween }} \\
\mathrm{mN} \mathrm{m}^{-1}\end{array}$ \\
& $\mathrm{~kg} \mathrm{~m}^{-3}$ & $\mathrm{~nm}$ & $\mathrm{~nm}$ & $\%$ & $\%$ & & $\mathrm{mN} \mathrm{m}^{-1}$ & $\mathrm{~m}^{2}$ \\
\hline APG 311 & $950 \pm 50$ & $8.2 \pm 0.03$ & - & $2.2 \pm 4.10^{-3}$ & - & $0.151 \pm 0.001$ & $24.7 \pm 0.8$ & $22.8 \pm 0.8$ & $5.9 \pm 0.6$ \\
EMG 900 & $1720 \pm 90$ & $11.3 \pm 0.1$ & $5 \pm 0.1$ & $9.2 \pm 0.1$ & $3.3 \pm 0.1$ & $1.67 \pm 0.04$ & $22 \pm 0.5$ & $29.2 \pm 1.4$ & $7 \pm 0.3$ \\
Magoil & $970 \pm 50$ & - & - & - & - & $4.7 \times 10^{-4} \pm 0.5 \times 10^{-4}$ & - & $5.8 \pm 0.4$ & - \\
\hline
\end{tabular}




\section{Extended Data Table E2}

Cell counts of whole blood from a control experiment, after pumping by Qpump, and after peristaltic pumping measured by haematology analyser (Sysmex XN-1000). Each set of experiments was performed using blood from the same donor. Abbreviation. RBC: Red Blood Cells. HGB: Haemoglobin. HCT: Haematocrit (volume percentage of red blood cells). MCV: Mean corpuscular volume (average red blood cell volume). MCH: Mean Corpuscular Haemoglobin (average quantity of haemoglobin per one red blood cell). MCHC: Mean Corpuscular Haemoglobin Concentration. RDW: Red blood cell distribution width. PLT: platelet counts. PDW: Platelet distribution width. MPV: Mean platelet volume. P-LCR: Platelet larger cell ratio. PCT: Plateletcrit (volume percentage of platelets in the blood). IPF: Immature platelet fraction. WBC: White blood cells.

\begin{tabular}{|c|c|c|c|c|c|c|c|c|c|c|c|c|c|c|c|}
\hline unit & $\begin{array}{c}\text { RBC } \\
10 \% \mu \mathrm{L} \\
\end{array}$ & $\begin{array}{l}\text { HGB } \\
\mathrm{g} / \mathrm{dL} \\
\end{array}$ & $\begin{array}{c}\mathrm{HCT} \\
\% \\
\end{array}$ & $\begin{array}{c}\text { MCV } \\
\mathrm{fL} \\
\end{array}$ & $\begin{array}{c}\mathrm{MCH} \\
\mathrm{Pg} \\
\end{array}$ & $\begin{array}{c}\mathrm{MCHC} \\
\mathrm{g} / \mathrm{dL}\end{array}$ & $\begin{array}{c}\text { RDW-SD } \\
\mathrm{fL} \\
\end{array}$ & $\begin{array}{c}\text { RDW-CV } \\
\% \\
\end{array}$ & $\begin{array}{l}\text { PLT\&F } \\
10^{3} / \mu \mathrm{L}\end{array}$ & $\begin{array}{c}\text { PDW } \\
\mathrm{fL} \\
\end{array}$ & $\begin{array}{c}\text { MPV } \\
\mathrm{fL} \\
\end{array}$ & $\begin{array}{c}\text { P-LCR } \\
\% \\
\end{array}$ & $\begin{array}{c}\text { PCT } \\
\% \\
\end{array}$ & $\begin{array}{l}\text { IPF } \\
\% \\
\end{array}$ & $\begin{array}{c}\text { WBC } \\
10^{3} / \mu \mathrm{L} \\
\end{array}$ \\
\hline ctrl & 4.07 & 12.3 & 35.5 & 87.2 & 30.2 & 34.6 & 42.8 & 13.2 & 203 & 11.6 & 10.5 & 28.2 & 0.21 & 3.3 & 3.58 \\
\hline Qpump 1 & 4.11 & 12.3 & 35.7 & 86.9 & 29.9 & 34.5 & 42.1 & 13.2 & 169 & 10.8 & 10.2 & 26.8 & 0.17 & 3.3 & 3.44 \\
\hline Qpump 2 & 3.99 & 12 & 34.7 & 87 & 30.1 & 34.6 & 41.4 & 13.1 & 159 & 11.4 & 10.5 & 26.9 & 0.17 & 3.3 & 3.46 \\
\hline Peristaltic pump 1 & 3.56 & 10.7 & 30.4 & 85.4 & 30.1 & 35.2 & 40.6 & 13.1 & 192 & 11.9 & 10.7 & 29 & 0.22 & 4.2 & 3.84 \\
\hline Peristaltic pump 2 & 4.06 & 12.3 & 34.7 & 85.5 & & & & & & & & & & & \\
\hline
\end{tabular}




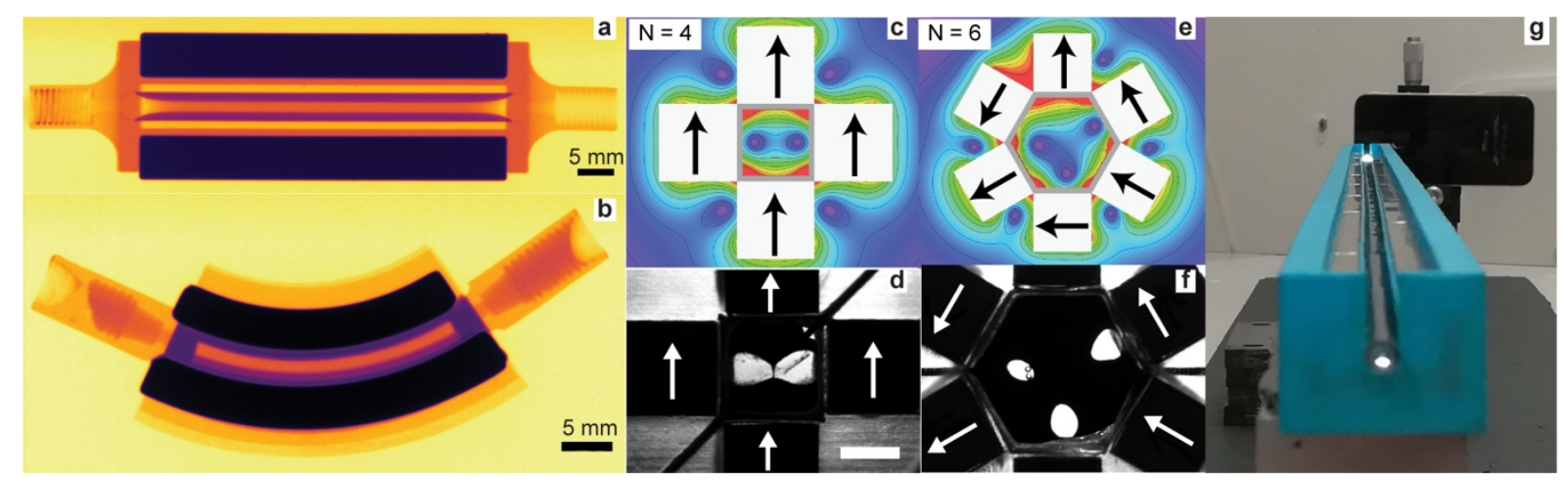

\section{Extended Data Fig. E1}

X-ray transmission images of an antitube of water inside a ferrofluid for a $6 \times 6 \times 50 \mathrm{~mm}$ cuboid magnets and $6 \mathrm{~mm}$ gap; and $\mathbf{b}$ matching arc magnets of height $20 \mathrm{~mm}, 3.5 \mathrm{~mm}$ gap, and inner and outer diameter pairs of: ID $25 \mathrm{~mm}$ OD $28.5 \mathrm{~mm}$, and ID $33 \mathrm{~mm}$ OD $36.5 \mathrm{~mm}$. Antitube cross-sections using non-quadrupolar fields: for a 4-magnet arrangement $\mathbf{c}$ magnetic field calculation, $\mathbf{d}$ experimental crosssection; and a 6-magnet arrangement e magnetic field calculation, $\mathbf{f}$ experimental cross-section. Scale bar is $3 \mathrm{~mm}$ (see videos 5,6$)$. $\mathbf{g}$ Side-view of a $1 \mathrm{~m}$ long water antitube $(d=2 \mathrm{~mm})$, which allows light to pass throughout to show the continuous water phase 
<smiles>O=C(O)CN(CCN1CC(=O)OC(=O)C1)CCN1CC(=O)OC(=O)C1</smiles>

DTPA dianhydride

Paramagnetic Oil
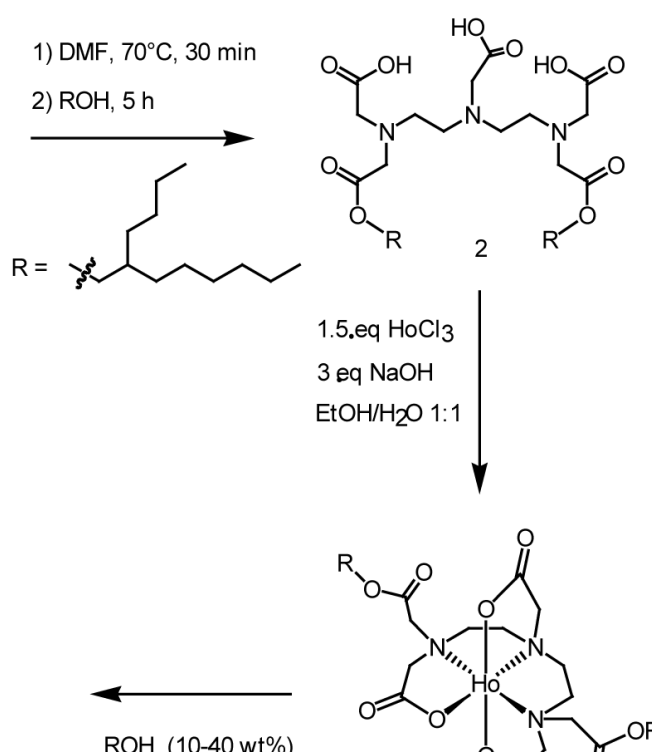

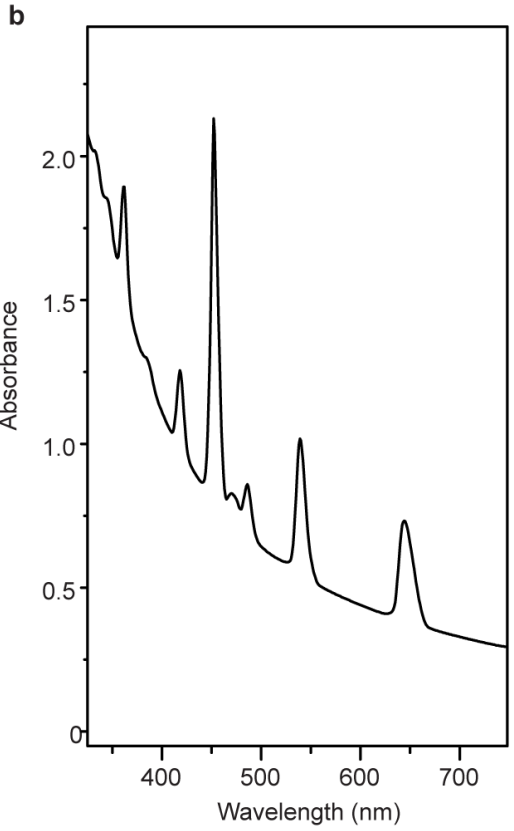

\section{Extended Data Fig. E2}

a Magoil synthesis reaction scheme. DMF (dimethylformamide), EtOH (Ethanol); b Absorption spectrum of $\mathrm{Ho}^{3+}$ based paramagnetic oil. 

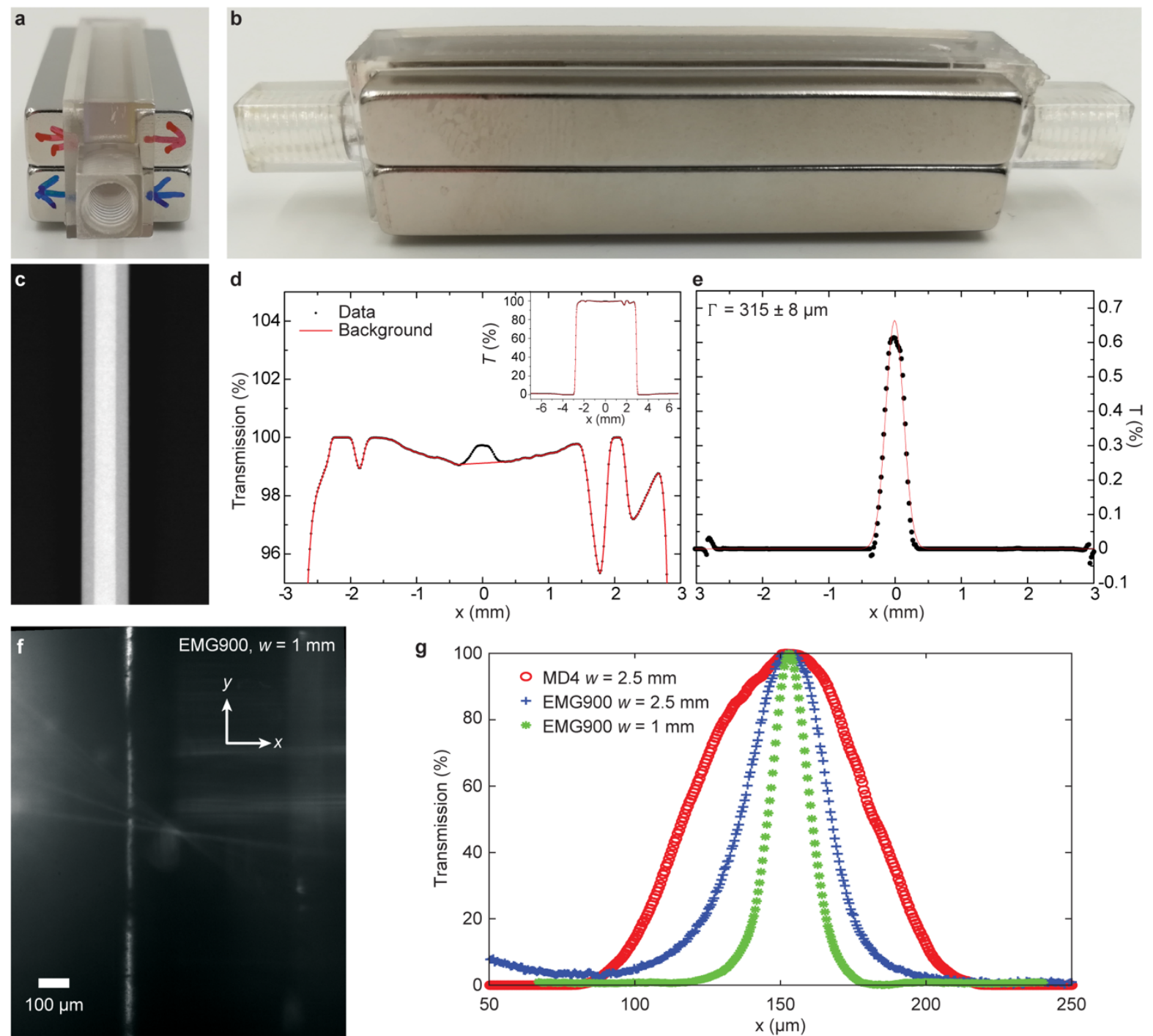

\section{Extended Data Fig. E3}

a \& b Typical quadrupole assemblies used for X-ray measurements; c inverted transmission X-ray image; d transmission averaged along the channel; e background corrected transmission through the water anti-tube fitted with a Gaussian peak function. f Optical image of a sub 100-micron water antitube in an EMG900 ferrofluid (double surfactant). $\mathbf{g}$ Intensity profile across the microfluidic channel in the vicinity of water antitube. The profile is column averaged along the length of microfluidic channel. 

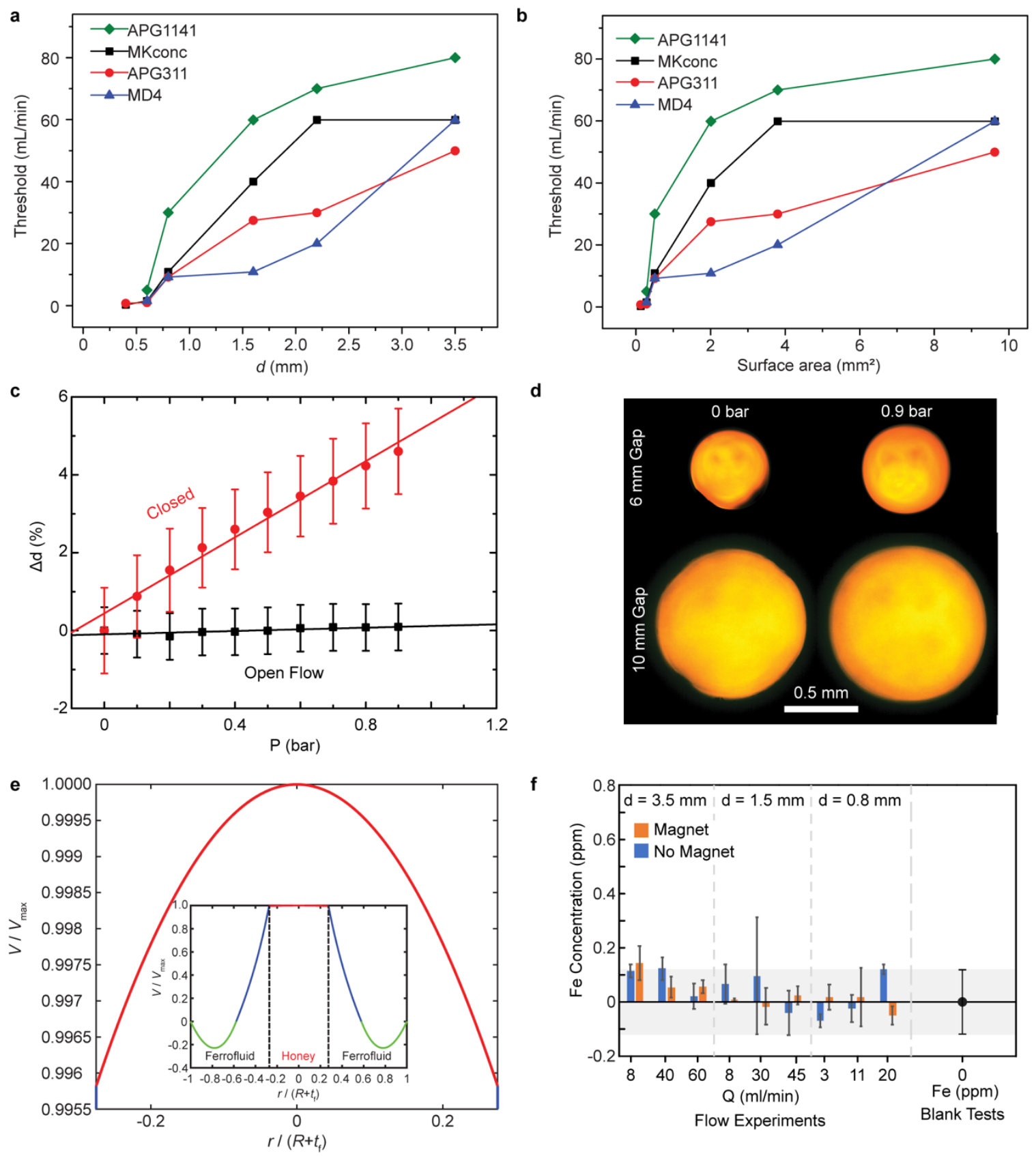

\section{Extended Data Fig. E4}

The threshold flow rate for antitube $\mathbf{a}$ diameters, and $\mathbf{b}$ areas; $\mathbf{c}$ relative dilation of an antitube in APG311 ferrofluid with a quadrupolar gap, $w$, of $10 \mathrm{~mm}$ under flow and with the outlet closed; d side view through the antitubes under static conditions with no flow (outlet closed) for two magnet gaps at equilibrium ( 0 bar) and under 0.9 bar pressure; e normalised velocity profile inside honey using eqns. $(1,2)$, corresponding to data in Fig. 2 d, inset shows velocity profile in both honey and ferrofluid; and $\mathbf{f}$ concentration of $\mathrm{Fe}$ found in water after pumping through three different antitube diameters for three flow rates with and without an extra magnet on the outlet flow. Blank tests for pure water give the grey background threshold detection. Values are averages of 6 samples, error bars are standard deviations. 

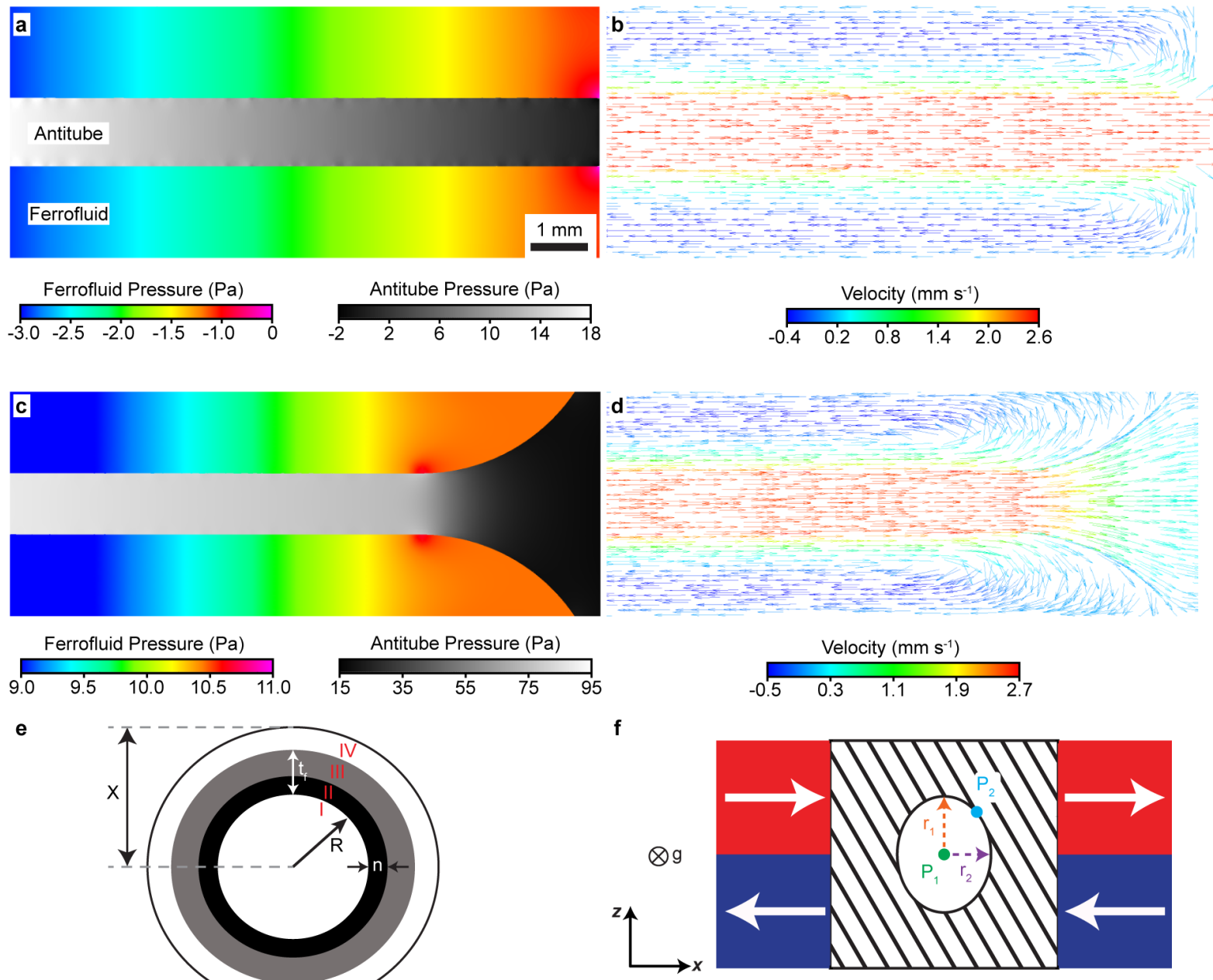

\section{Extended Data Fig. E5}

Contour plots from numerical simulations of a honey antitube in EMG900 ferrofluid under $175 \mu \mathrm{l} / \mathrm{min}$ flow rate for two cases, first the semi-infinite case with no inlet effects $(\mathbf{a}, \mathbf{c})$, second the finite case including inlet effects with the ferrofluid contours matching those found by experiment (b,d), c.f. Fig. E1a. The plots are a,b isometric pressure contours for at the outlets, note the different colourmaps for the pressure inside the ferrofluid versus inside the antitube; c, d velocity vector field at the outlets. Geometries used in the derivation of equations 1-4: e Cross section of liquid tube system considered in M19; with four different flow regions consisting of I honey, II a parallel flow of ferrofluid, III a counterflow of ferrofluid, and IV a fictitious region to define the radial distance $x$ at which the flow velocity becomes zero. Thus, the slip length for a flow of honey is $b=x-R, t_{f}$ is the thickness of the ferrofluid, $n$ is the thickness of region with shear flow, and $R$ is radius of the honey tube; $\mathbf{f} 2 \mathrm{D}$ geometry of 4 bar magnets in a quadrupolar configuration considered in M20, the hatched region denotes the ferrofluids, while the white region in the centre is the contained liquid tube. 

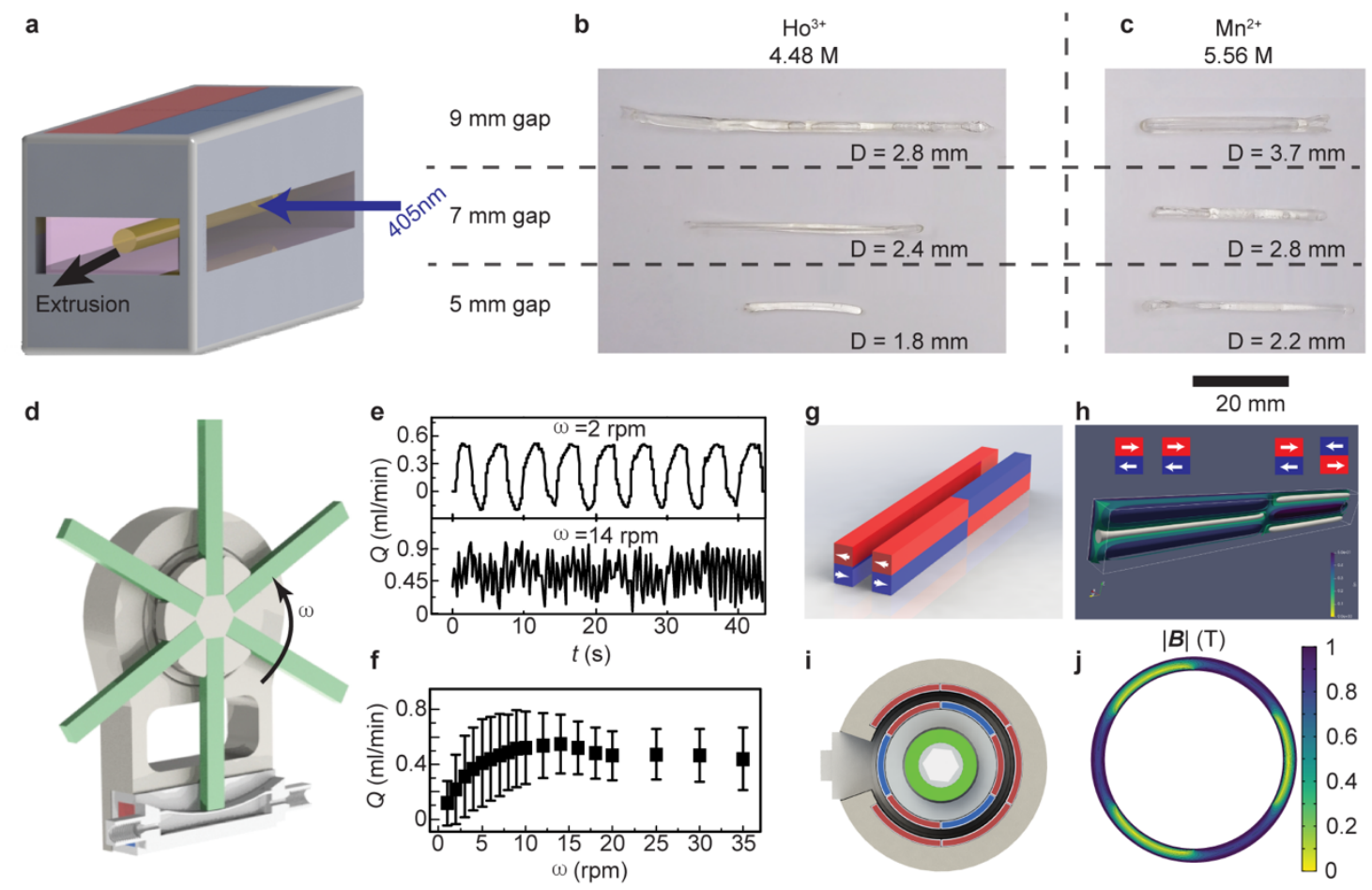

\section{Extended Data Fig. E6}

a A scheme of the fluidic chip used for the extrusion of a photopolymer resin and its photopolymerization by $405 \mathrm{~nm}$ laser during extrusion. Photos of polymerized tubes extruded in $\mathbf{b}$ an aqueous $\mathrm{HoCl}_{3}$ solution, and $\mathbf{c}$ an aqueous $\mathrm{MnCl}_{2}$ solution. The diameter of the tubes decreased as the magnet gap decreased, and those photopolymerised in $\mathrm{Ho}^{3+}$ were smaller as $\mathrm{Ho}^{3+}$ has higher magnetic susceptibility than $\mathrm{Mn}^{2+}$. d Isometric-view of a 6-spoke magnetostaltic pump (cf. video 11); e Slow rotation $(2 \mathrm{rpm})$ leads to pulsed flow, while fast rotation $(14 \mathrm{rpm})$ produces a smoother flow; $\mathbf{f}$ the average flow rate and standard deviation vs. rotation rate $\omega$. $\mathbf{g}$ The magnetic configuration for an antitube (first half) and a pinching region (second half) using 6x6x50 mm magnets with the gap of 6 $\mathrm{mm} ; \mathbf{h}$ isosurface plot of its calculated magnetic field. The weak field region where water can reside is highlighted by a white colour. A water antitube created by the quadrupolar arrangement (left half) is disrupted at the interface between two regions. The field created by the pinching geometry has a field strength of $0.5 \mathrm{~T}$ at the centre of magnets (represented by the purple colour); i Top-view of the Qpump based on this principle. The orientation of magnetization for the arc segments is radially outward (red) or inward (blue); $\mathbf{j} x-y$ contour plot of the calculated magnetic field along the $z$-axis centre of the Qpump. 


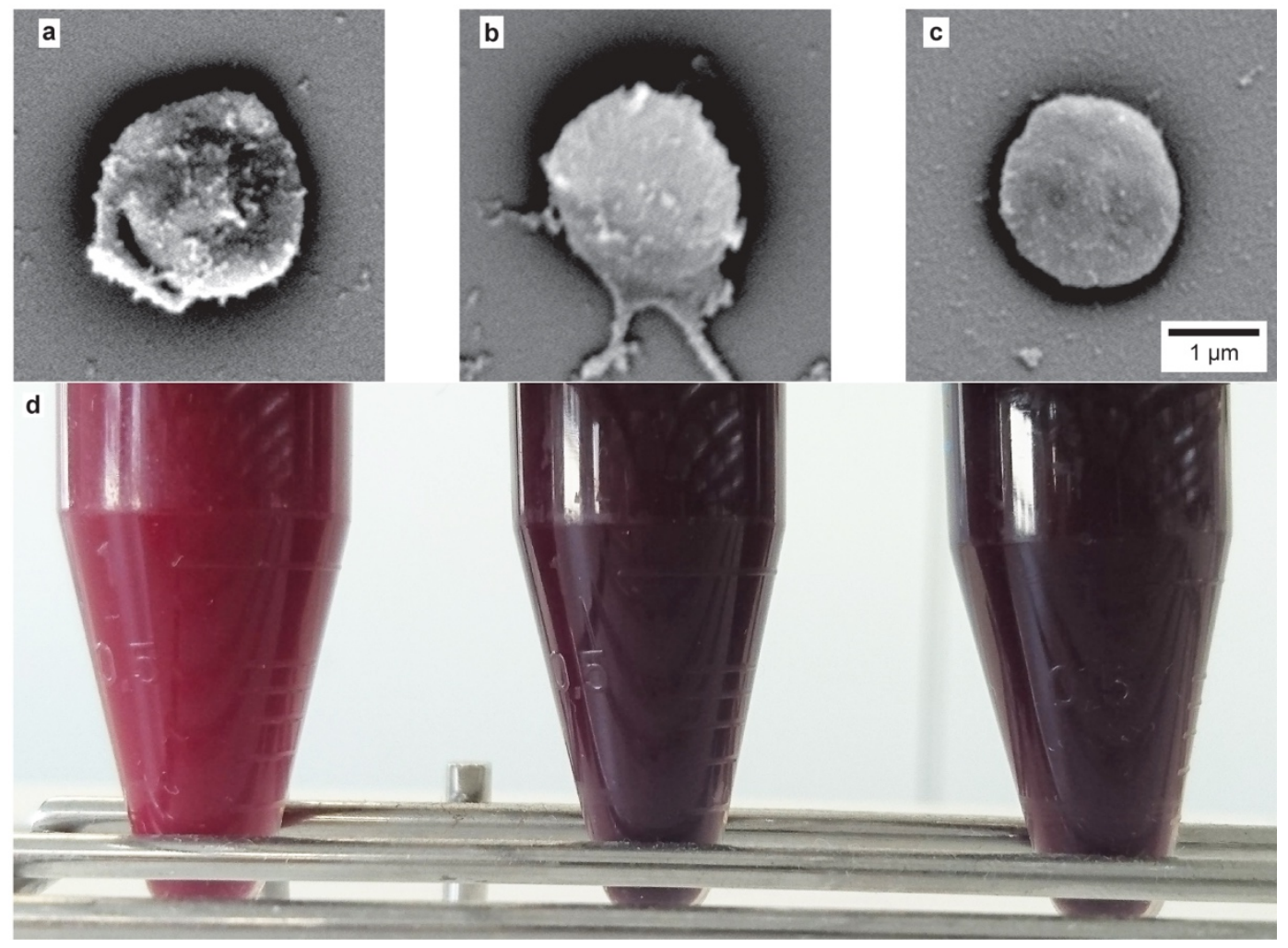

\section{Extended Data Fig. E7}

Representative scanning electron microscopy (SEM) images of platelets, a after peristaltic pumping; b platelets from control blood; and c platelets from blood after Qpump pumping. No major morphological change due to the activation of platelets was observed for both types of pumps (i.e., peristaltic and Qpump). d A picture of the whole blood in a tube after peristaltic pumping (left), control (middle) and Qpump pumping (right). 


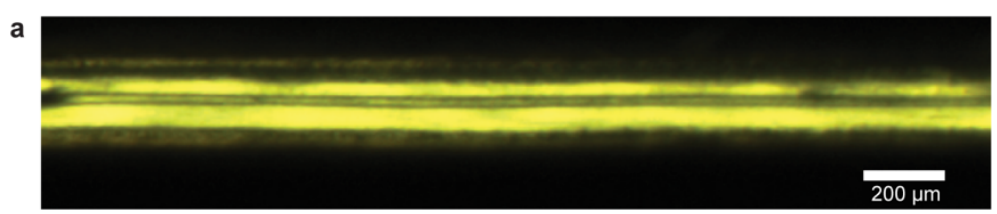

b
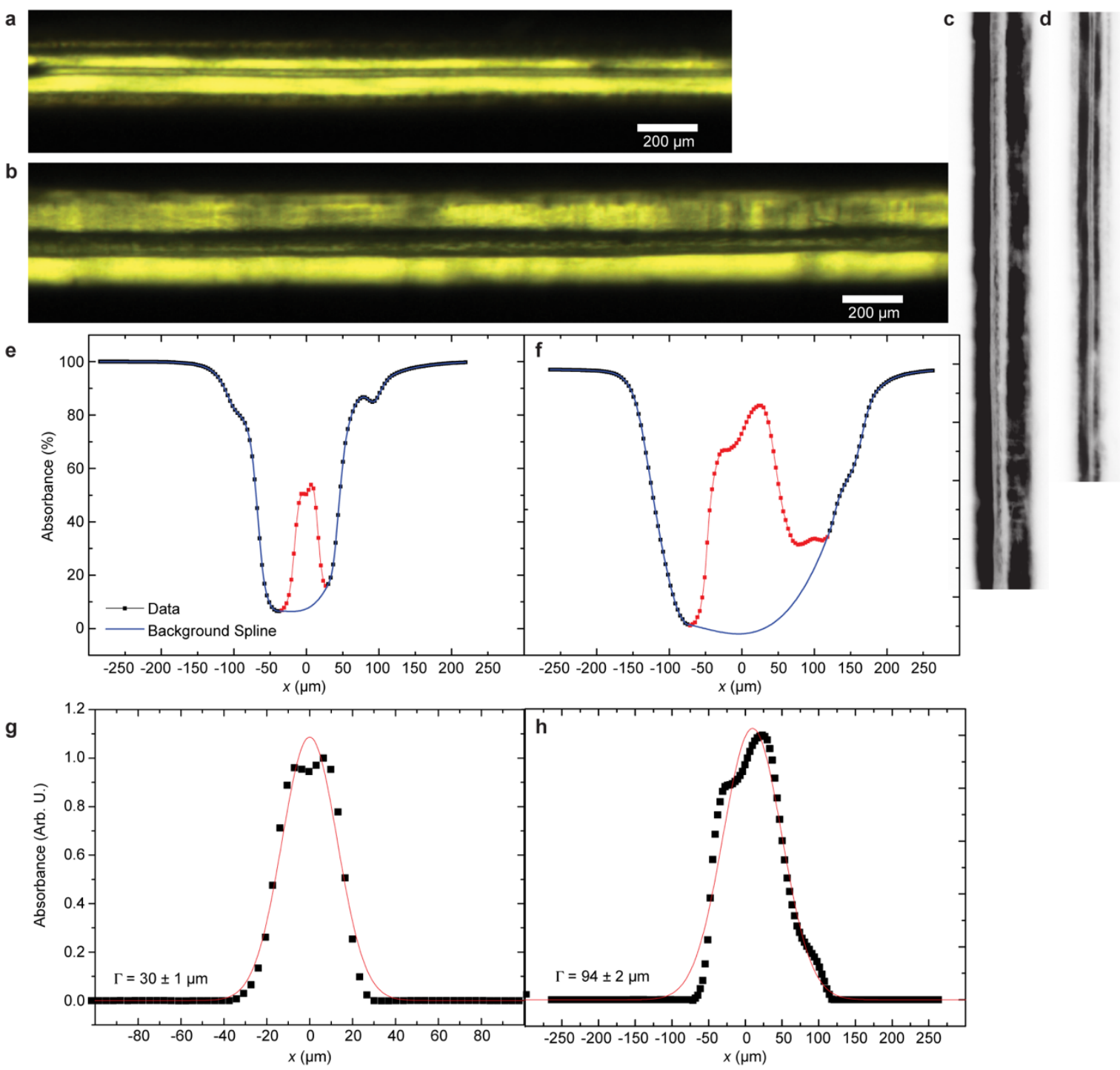

\section{Extended Data Fig. E8}

Optical micrographs of a water tube in Magoil for a gap width $\mathbf{a} w=220 \mu \mathrm{m}$, and $\mathbf{b} w=307 \mu \mathrm{m}$; $\mathbf{c} \&$ $\mathbf{d}$ are the greyscale, rotated and inverted images, $\mathbf{e} \& \mathbf{f}$ are the column average profiles of each gap, and $\mathbf{g} \& \mathbf{h}$ are the Gaussian function fits to the background subtracted profiles. Note: the $94 \mu \mathrm{m}$ antitube is thermodynamically stable, as the image was taken during the extrusion of the water antitube, while the $30 \mu \mathrm{m}$ tube is thermodynamically unstable. After injection, water was then extracted, resulting in a thinning of the tube, which at this diameter collapses into droplets in a matter of minutes. 


\section{Supplementary Text}

Additional information is provided in supplementary information in the following sections:

I. Langevin and demagnetisation expressions used to correctly determine the magnetic properties of the ferrofluid (M4)

II. Expressions used to determine the remnant magnetisation of the permanent magnets (M6)

III. Full derivations for equations 1 and 2 (M18)

IV. Derivation of Equation 3 (M19)

V. Derivation of equation 4 (M20)

\section{Video captions}

\section{Video 1}

Extrusion and extraction of water into Holmium based Magoil in a quadrupolar field using two in/outlets. The water is dyed with ink to enhance visibility. Bubbles are removed from the top Magoilair interface manually.

\section{Video 2}

Rupture of an anti-tube in Magoil by a plastic stick, which self-heals without any flow from an external pump.

\section{Video 3}

Photocrosslinking of photopolymer resin that was extruded into Holmium aqueous solution in a quadrupolar arrangement of magnets.

\section{Video 4}

Honey flowing from reservoirs under gravity through three different configurations: an antitube, no tube (i.e., air only), and a normal tube of the same diameter.

\section{Video 5}

Injection of APG311 ferrofluid into a water-containing thin cell surrounded by four $10 \times 10 \times 10 \mathrm{~mm}^{3}$ cube magnets with the field orientation shown in Fig. E1d. 


\section{Video 6}

Injection of APG311 ferrofluid into a water-containing thin cell surrounded by six 10x10x10 mm cube magnets with the field orientation shown in Fig. E1f.

\section{Video 7}

Splitting of water through a free-hanging Y-junction in a ferrofluid. The ferrofluid is held in place by the magnetic field gradient force supplied by the magnets, there is no physical support below it. Water is injected from one input on the left and splits in two on the right.

\section{Video 8}

Valving an antitube in ferrofluid using a fifth magnet; upon addition of a magnet the flow stops, upon removal, the flow recommences.

\section{Video 9}

Animated simulations of $0.15 \mathrm{~T}$ isovolume of the magnetic field in a quadrupolar field upon valving with one magnet.

\section{Video 10}

Animated simulations of the $0.15 \mathrm{~T}$ isovolume of the magnetic field in a quadrupolar field upon valving with two magnets.

\section{Video 11}

Non-contact peristaltic pumping using moving valve-points (occlusions) generated by a rotating wheel of valving magnets.

\section{Video 12}

The demonstration of pumping water by a Qpump. The outlet was connected to a pressure sensor to measure the pressure generated, showing $\sim 900$ mbar of pumping pressure. 